\title{
Machine Translation using Semantic Web Technologies: A Survey
}

\author{
Diego Moussallem $^{\mathrm{a}, \mathrm{b}, *}$, Matthias Wauer ${ }^{\mathrm{a}}$, Axel-Cyrille Ngonga Ngomo ${ }^{\mathrm{b}}$ \\ ${ }^{a}$ University of Leipzig \\ AKSW Research Group \\ Department of Computer Science \\ Augustusplatz 10, 04109 Leipzig, Germany \\ ${ }^{b}$ University of Paderborn \\ Data Science Group \\ Pohlweg 51, D-33098 Paderborn, Germany
}

\begin{abstract}
A large number of machine translation approaches have recently been developed to facilitate the fluid migration of content across languages. However, the literature suggests that many obstacles must still be dealt with to achieve better automatic translations. One of these obstacles is lexical and syntactic ambiguity. A promising way of overcoming this problem is using Semantic Web technologies. This article presents the results of a systematic review of machine translation approaches that rely on Semantic Web technologies for translating texts. Overall, our survey suggests that while Semantic Web technologies can enhance the quality of machine translation outputs for various problems, the combination of both is still in its infancy.
\end{abstract}

Keywords: machine translation, semantic web, ontology, linked data, multilinguality, knowledge graphs

\section{Introduction}

Alongside increasing globalization comes a greater need for readers to understand texts in languages foreign to them. For example, approximately $48 \%$ of the pages on the Web are not available in English 1 . The technological progress of recent decades has made both the distribution and access to content in different languages ever simpler. Translation aims to support users who need to access content in a language in which they are not fluent [1, 2].

However, translation is a difficult task due to the complexity of natural languages and their structure [3. In addition, manual translation does not scale to the magnitude of the Web. One remedy for this problem is Machine Translation (MT). The main goal of MT is to enable people to assess content in languages other than the languages in which they are fluent [4]. From a formal point of view, this means that the goal of MT is to transfer the semantics of text from an input language to an output language [5]. At the time of writing, large information portals such as Google $\mathrm{f}^{2}$ or Bing $\mathrm{g}^{3}$ already offer MT services that are widely used.

Although MT systems are now popular on the Web, they still generate a large number of incorrect translations. Recently, Popović [6] has classified five types of errors that still

\footnotetext{
* Principal corresponding author

Email addresses: moussal leme informatik.uni-leipzig.de (Diego Moussallem), wauerainformatik.uni-leipzig.de

(Matthias Wauer), axel.ngonga@upb. de (Axel-Cyrille Ngonga Ngomo)

1 https: / / www. internetworldstats.com/stats 7 .htm

${ }^{2}$ http://translate.google.com.br/about/

${ }^{3}$ http://www.bing.com/translator/help/
}

remain in MT systems. According to research, the two main faults that are responsible for $40 \%$ and $30 \%$ of problems respectively, are reordering errors and lexical and syntactic ambiguity. Thus, addressing these barriers is a key challenge for modern translation systems.

A large number of MT approaches have been developed over the years that could potentially serve as a remedy. For instance, translators began by using methodologies based on linguistics which led to the family of Rule-Based Machine Translation (RBMT). However, RBMT systems have a critical drawback in their reliance on manually crafted rules, thus making the development of new translation modules for different languages even more difficult 7,8 .

Statistical Machine Translation (SMT) and Example-Based Machine Translation (EBMT) were developed to deal with the scalability issue in RBMT [9], a necessary characteristic of MT systems that must deal with data at Web scale. Presently, these approaches have begun to address the drawbacks of rule-based approaches. However, some problems that had already been solved for linguistics based methods reappeared. The majority of these problems are connected to the issue of ambiguity, including syntactic and semantic variations [2].

Subsequently, RBMT and SMT have been combined in order to resolve the drawbacks of these two families of approaches. This combination of methods is called hybrid MT. Although hybrid approaches have been achieving good results, they still suffer from some RBMT problems [10-12], for example, the big effort of adding new rules for handling a given syntax divergence. Nowadays, a novel SMT paradigm has arisen called Neural Machine Translation (NMT) which relies on Neural Network (NN) algorithms. NMT has been achieving 
impressive results and is now the state-of-the-art in MT approaches. However, NMT is still a statistical approach sharing some semantic drawbacks from other well-defined SMT approaches 13.

One possible solution to address the remaining issues of MT lies in the use of Semantic Web Technologies (SWT), which have emerged over recent decades as a paradigm to make the semantics of content explicit so that it can be used by machines [14]. It is believed that explicit semantic knowledge made available through these technologies can empower MT systems to supply translations with significantly better quality while remaining scalable [15]. In particular, the disambiguated knowledge about real-world entities, their properties and their relationships made available on the Linked Data (LD) Web can potentially be used to infer the right meaning of ambiguous sentences or words and also to support the reordering task.

The obvious opportunity of using SWT for MT has already been studied by a number of approaches. This systematic survey gives an overview of existing systems making use of this combination and presents the difference in translation quality that they produce, especially w.r.t. the issue of ambiguity. Based on this overview, we distill the challenges and opportunities in the use of SWT in MT for translating texts.

This paper is structured as follows: In Section 2 , we describe the methodology used to conduct this systematic survey. Section 3 discusses different MT approaches and their particular challenges. Section 4 shows how SWT have been used in MT approaches and presents suggestions on how to handle the challenges. Section 5 concludes with ideas for future work.

\section{Research Method}

The research methodology behind this survey follows the formal systematic literature review process. In particular, this study is based on the guidelines proposed in [16-18]. As detailed below, we also took into account other surveys from relevant journals as well as surveys about related topics such as Word Sense Disambiguation (WSD) and Semantic Web (SW).

\subsection{Research Questions}

The goal of this survey is to provide SW researchers with existing methodologies that use SWT applied to MT systems for translating natural-language sentences. To achieve this goal, we aimed to answer the following general research question: How can SWT enhance MT quality? This question was then divided into four sub-questions as follows:

RQ1. What are state-of-the-art approaches in MT which use SWT?

RQ2. Which SWT are applied in MT?

RQ3. Does ontological knowledge influence the quality of an automatic translation?
RQ1 intends to collect available research works which retrieve knowledge from SW resources for translating texts. RQ2 aims to provide an explicit comparison among SWT used in each respective MT approach. RQ3 attempts to resolve whether inclusion of a certain concept represented or inferred by an ontology supports and improves the translation process of a given MT system. RQ4 asks for a description of all available SW tools that have been used and may be used in future work for supporting MT systems.

\subsection{Search Strategy}

An overview of our search methodology and the number of articles collected at each step is shown in Figure 1 and described in detail below. To start the search, it was essential to determine search criteria that fit the purposes of our survey. Based on best practices [16-18], we defined the following selection criteria to classify the retrieved studies.

\subsubsection{Inclusion criteria}

The papers considered in our study were publications in English between 2001 and 2017. They had to satisfy at least one of the following criteria:

- a focus on distinguishing between ambiguous words in MT that use SWT.

- proposed and/or implemented an approach for MT using SWT.

- contain a combination of ontological knowledge with MT for handling structural divergence issues.

\subsubsection{Exclusion criteria}

None of the criteria below was to hold for the papers considered in this survey:

- not peer-reviewed or published.

- assessment methodologies published as a poster abstract.

- no use of SWT in MT for translating natural language sentences.

- not proposing an MT approach or framework which retrieves information using SWT.

\subsubsection{Search queries}

To address the research questions and criteria, we determined a set of keyword queries that allowed us to detect relevant studies for our survey. We used the following keywords: machine translation, semantic web, ontology, linked data, disambiguation, methodology, and multilingual. The choice of these keywords corresponds to the main keywords used by SW and MT works in their titles. Also, the keywords multilingual and methodology were identified to return papers

RQ4. What kinds of SW driven tools are available for MT? 


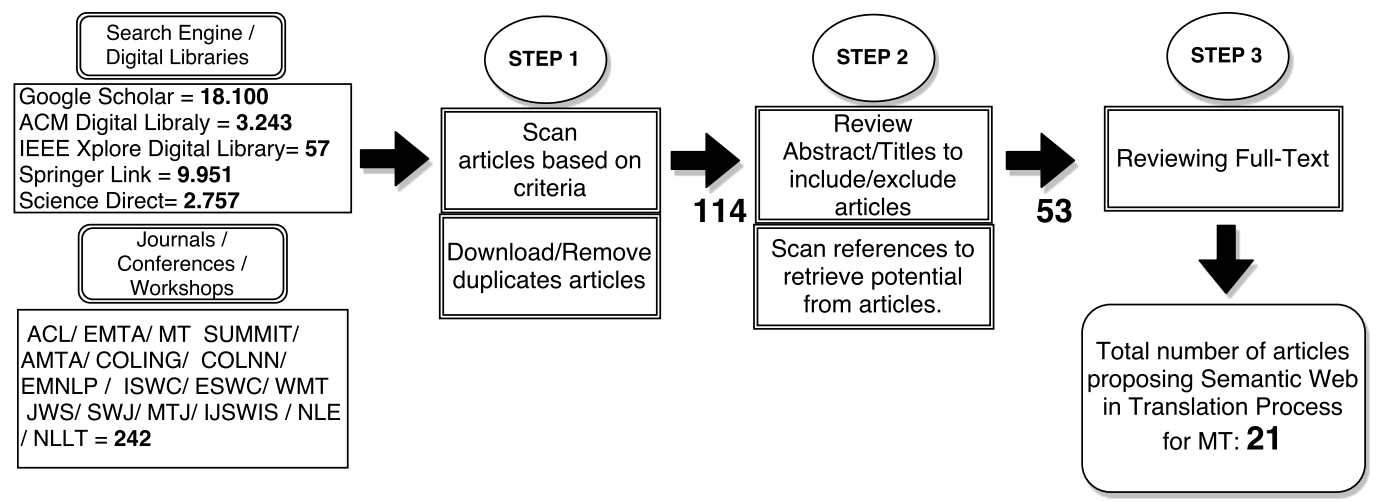

Figure 1: Steps followed to retrieve the systematic review results.

matching the inclusion criteria. Therefore, they were combined in two search queries in order to retrieve the relevant research works 4

1. machine translation AND (ontology OR linked data OR semantic web OR disambiguation)

2. (methodology OR multilingual OR disambiguation) AND (linked data OR ontology OR semantic web OR machine translation)

Thereafter, we used the following search engines, digital libraries, journals, conferences, and workshops to find relevant publications.

Search engines and digital libraries:

- Google Scholar ${ }^{5}$

- ACM Digital Library

- IEEE Xplore Digital Library 7

- SpringerLink 8

- ScienceDirect9

- MT-Archive 10

Journals:

- Semantic Web Journal (SWJ) 11

- Journal of Web Semantics (JWS) 12

- Machine Translation Journal (MT) 13

\footnotetext{
${ }^{4}$ The search engines automatically take into account the inflections and synonyms of the keywords.

5 http://scholar.google.com/

6 http://dl.acm.org/

7http://ieeexplore.ieee.org/

\&ttp://link.springer.com/

chttp://www.sciencedirect.com/

10 http://www.mt-archive.info/

11 http://www.semantic-web-journal.net/

12 http://www.websemanticsjournal.org/

13 http://link.springer.com/journal/10590
}

- Natural Language and Linguistic Theory

- Natural Language Engineering

- International Journal on Semantic Web and Information Systems (IJSWIS) ${ }^{16}$

Conferences and associated workshops:

- Association for Computational Linguistics (ACL) 1934

- North American Association for Computational Linguistics (NAACL) 35-45]

- Empirical Methods in Natural Language Processing (EMNLP) [46-59]

- International Conference on Computational Linguistics (COLING) 60-67

- International Association for Machine Translation (IAMT, AMTA, EAMT, MT Summit) 17

- World Wide Web Conference (WWW) 68-84

- International Semantic Web Conference (ISWC) 8598, 98,-100

- Extended Semantic Web Conference (ESWC) 101-114

\subsection{Search Steps}

The initial search based on the queries described above returned 34,350 publications. The subsequent processing of this set of publications was divided into three steps.

\footnotetext{
$1 4 \longdiv { \text { http://link.springer.com/journal/11049 } }$

15 http://journals.cambridge.org/action/ displayJournal?jid=NLE

ichttp://www.ijswis.org/

17 http://www.mt-archive.info/srch/conferences-1.htm
} 


\subsubsection{Step 1}

This step ensures that the publications considered in the study abide by the selection criteria. Hence, we excluded publications that are not in English or do not contain any reference to SW and MT. Moreover, we excluded all publications that were not peer-reviewed, as well as works that were published as posters or abstracts. We manually scanned the articles based on the criteria presented above.

Thereafter, the bibliographic metadata of remaining publications was analyzed using the bibliography management platform Mendeley ${ }^{18}$ and 21 duplicates were removed.

\subsubsection{Step 2}

For this step, we reviewed the abstracts of the 114 articles returned by Step 1 based on the four research questions described in Section 2.1. 64 of the 114 articles were excluded because they did not SWT. However, all 114 articles were documented as a reference source for potential future articles. To retrieve articles from references we adopted the following strategy:

1. We searched for the article title in Google Scholar and retrieved the "Cited By" articles.

2. We then read the abstract of each potential article found by searching the references of all 50 articles and by these means, found 3 more articles.

\subsubsection{Step 3}

With a total of 53 articles deemed potentially relevant for the survey, we read all articles completely to evaluate their suitability for our study. As a final result, we selected 21 articles published between 2001 and 2017, which are listed in Table 1 10 of these 21 articles matched the criteria, which propose and/or implement MT using SWT. 6 of 21 matched the criteria for disambiguation processes in MT using SWT. 5 of 21 matched the criteria that focused on combining ontological knowledge with MT to handle syntactic divergences. Most of the remaining relevant articles are related to the translation of ontology labels. Although ontology label translation is an important task relevant to some of the articles, we do not consider them in this survey because ontology labels are single words or compounds rather than sentences.

\section{Classification of MT Approaches}

In this section, we give an overview of generic dimensions across which MT systems can be classified. This overview allows for a better understanding of the approaches retrieved as described above. A detailed description of the approaches is given in Section 3.2. Sequentially, we introduce the remaining dimensions. Afterwards, we present the open MT challenges pertaining to all MT approaches. Finally, we briefly introduce common MT evaluation metrics in order to provide a background to how MT systems are evaluated automatically.

18 https://www.mendeley.com/groups/7405201/ semantic-web-machine-translation/papers/

\subsection{Dimensions}

We classify MT systems across three dimensions.

1. Architecture: From an architectural perspective, it is assumed that all MT paradigms could be subsumed under one architecture to model existing MT systems [2]. However, the architecture may be composed of more than one approach and these approaches depend, for their operation, on the amount of available knowledge. For example, some approaches rely purely on statistics (SMT) while others use complex linguistic models (RBMT) to compute a translation.

2. Problem space addressed: Previous works (e.g., [136]) suggest that particular MT approaches are best suited to address particular types of problems. For instance, an approach for translating old Egyptian texts should rely on deep linguistics rules due to the lack of bilingual corpora. In contrast, translating large volumes of text is best carried out using statistics models because resolving many errors from hand-crafted rules requires a big human effort. Furthermore, the usage of statistics will depend on the language and availability of bilingual corpora for training.

3. Performance: A central challenge of MT is to create realtime MT solutions that achieve a high-quality translation of variable-nature texts, while being low in complexity to build. For example, while SMT performs well on long texts (paragraphs containing 100 words), it often fails on short sentences ( e.g., social network comments and subtitles) - mainly when a given SMT is built using large corpora and needs to translate text from different domains [2].

\subsection{Architectures}

Currently, the architectures of MT systems are subdivided into RBMT, SMT and EBMT. In addition, hybrid systems which combine RBMT with SMT have emerged over recent years. Figure 2 gives an overview of a generic MT architecture that may represent the workflow of all approaches. The left side of the triangle comprises generically of source text analysis, while the right side corresponds to the generation of target texts. Both sides of this generic architecture have four main steps. (1) A morphological step, which handles the morphology of words. (2) A syntactic step, which deals with the structure of sentences. (3) A semantics' step, which considers the meaning of words and sentences. Finally, (4) an interlingual phase, which may be seen as a generic representation of source and target text either in RBMT (internal model) or SMT approaches (statistical model).

\subsubsection{Rule-Based Machine Translation}

At a basic level, RBMT approaches carry out a translation in the following manner: first, they parse the input text. Then they create an intermediary linguistic representation of the scanned text. They finish by generating text in the target language based on morphological, syntactic, and semantic mappings between the two languages. RBMT approaches 
Table 1: List of the selected papers.

\begin{tabular}{|c|c|}
\hline Citation & Title \\
\hline C. Vertan 115 & Language Resources for the Semantic Web - perspectives for Machine Translation \\
\hline W. Hahn and C. Vertan 116 & Challenges for the Multilingual Semantic Web \\
\hline N. Elita and A. Birladeanu [117] & A First Step in Integrating an EBMT into the Semantic Web \\
\hline C. Shi and H. Wang 118 & Research on Ontology-driven Chinese-English Machine Translation \\
\hline N. Elita and M. Gavrila 119 & Enhancing translation memories with semantic knowledge \\
\hline E. Seo et al. 120 & Syntactic and Semantic English-Korean Machine Translation using Ontology \\
\hline P. Knoth et al. [121] & Facilitating Cross-Language Retrieval and Machine Translation by Multilingual Domain Ontologies \\
\hline L. Lesmo et al. [122] & An Ontology based Architecture for Translation \\
\hline A. M. Almasoud and H. S. Al-Khalifa [123] & A Proposed Semantic Machine Translation System for Translating Arabic Text to Arabic Sign Language \\
\hline \begin{tabular}{ll|l|l|} 
B. Harriehausen-Mühlbauer and T. Heuss & 15 & 124 \\
\end{tabular} & Semantic Web based Machine Translation \\
\hline K. Nebhi et al. [125] & NERITS - A Machine Translation Mashup System using Wikimeta and Linked Open Data \\
\hline J. P. McCrae and P. Cimiano 126 & Mining Translations from the Web of Open Linked Data \\
\hline D. Moussallem and R. Choren [127] & Using Ontology-based Context in the Portuguese-English Translation of Homographs in Textual Dialogues \\
\hline O. Lozynska and M. Davydov 128 & Information technology for Ukrainian Sign Language translation based on Ontologies \\
\hline K. Simov et al. 129 & Towards Semantic-based Hybrid Machine Translation between Bulgarian and English \\
\hline T.S. Santosh Kumar. [130] & Word Sense Disambiguation Using Semantic Web for Tamil to English Statistical Machine Translation \\
\hline N. Abdulaziz et al. 131 & Towards an Arabic-English Machine-Translation Based on Semantic Web \\
\hline J. Du et al. 132 & Using BabelNet to Improve OOV Coverage in SMT \\
\hline A. Srivastava et al. 133 & How to Configure Statistical Machine Translation with Linked Open Data Resources \\
\hline C. Shi et al. 134 & Knowledge-based Semantic Embedding for Machine Translation. \\
\hline A. Srivastava et al. 135 & Improving Machine Translation through Linked Data. \\
\hline
\end{tabular}

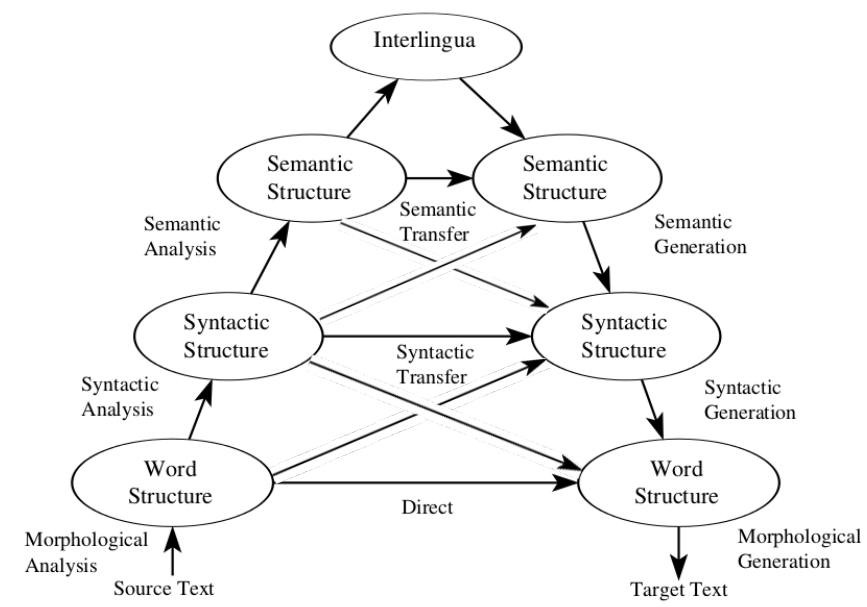

Figure 2: Generic MT Architecture - Vauquois triangle

can be divided into three categories: direct, transfer-based, and interlingua-based. In order to perform well, all of these approaches require extensive lexicons and large sets of rules designed by professional linguists.

3.2.1.1. Direct approaches. This family emerged with the first MT created by IBM [137]. Another translator which implemented this idea was Systran [138. Commonly, direct approaches are word-based or dictionary-based, translating the words one by one. Hence, they translate the texts without considering the meaning variations in the words, which leads to a significant error rate. Thus, the direct translation is seen as the first step in modern MT systems and since it can easily be combined with other technologies, it is commonly used by hybrid MT systems.

3.2.1.2. Transfer-based approaches. Transfer-Based Machine Translation (TBMT) was created due to the obvious necessity of preserving meaning whilst translating. TBMT has three steps: analysis, transfer, and generation. In the first step, TBMT systems analyze the sentence structure of the source language and generate an internal representation based on linguistic patterns of the target language. Subsequently, TBMT systems translate only the text for both respective languages. To this end, they use three dictionaries during the translation process: two monolingual dictionaries (source language and target language) and a bilingual dictionary containing a mapping between the source and the target languages [139].

3.2.1.3. Interligua-based approaches. The interlingual approach relies on similar insights to those underlying TBMT. The main difference between them is the internal model that they rely upon. In contrast to TBMT approaches (where the target language plays a key role), Interlingua-based approaches rely on extracting a language-independent representation of the input text. Hence, this can be easily adapted to translation into multiple target languages. State-of-the-art interlingual approaches rely most commonly on morphological, 
syntactic, and semantic rules based on a grammatical framework [140]. These are more accurate than comparable previously mentioned approaches (i.e., direct and TBMT). In addition, a direct beneficial consequence of the interlingual approach is the time-efficient portability of its framework to other languages. This approach is widely used by linguists for morphologically rich languages because it allows them to choose the better language representation of a translation model based on the source text. On the other hand, to achieve a highquality translation with Interlingua, the operator must carry out the translation within a specific domain.

\subsubsection{Statistical Machine Translation}

SMT relies upon the intuition that RBMT shortcomings can be addressed using experience. Commonly, SMT approaches acquire this experience from previous translations. These previous translations are collected from bilingual corpora, either parallel or comparable. They are crucial for the performance of SMT approaches. A bilingual parallel corpus is comprised of a source text and its respective translation. In contrast, a comparable corpus does not contain an exact translation of a source text, but a similar corpus in another language which may share the same topic, size or given time. SMT approaches heavily depend on Machine Learning (ML) techniques including supervised, unsupervised, and semi-supervised algorithms to compute a statistical translation model from the input corpus for use in subsequent translations. Most commonly, the goal of SMT approaches is simply to detect the translation that achieves the maximum probability or score for a given translation pair. SMT requires well-suited parallel or comparable bilingual corpora to achieve high accuracy. Comparable corpora can be used to get more terminological knowledge into a certain SMT system, but the SMT still needs an underlying language model built on parallel data. Hence, the translation quality directly depends on the language model trained on a given target side of the parallel corpus, which is a subset of the respective bilingual corpora.

Every SMT system shares at least three common steps. First, an alignment between the parallel or comparable corpora is necessary. Both source and target corpora are aligned in order to provide a mapping between each respective translation. When parallel corpora do not exist, there are ways to handle comparable corpora using algorithms that take alignment probabilities into account. Although it may affect the quality of a given SMT, this is a common task when there are insufficient reference translations. Second, a translation model needs to be created and trained over the alignment previously done, thus being responsible for translating the content from source to target language. At training phase, an underlying model is created which contains statistical frequencies of words and may contain syntactic information (from a hierarchy model or manually inserted) about both languages. Once the model has been created, the source text can be processed by using a function akin to a probability or score distribution contained in the model. Afterward, the step called decoding is essentially a search function that accesses the translation model by getting all the translation candidates and pro- viding a ranked list of possible translations. The language model which is created from a reference translation usually made by humans guarantees the fluency of a certain target language [2]. The following five sub-approaches exist in SMT.

Word-based - This approach was the first statistical one created by IBM which contains at least five IBM models. The Word-based approach basically translates one word at a time based on its frequency computed by the translation model over the entire training data. By ignoring multiple meanings of a given word (e.g., polysemous or homonymous words), the approach can generate wrong translations. Considering this weakness, Yamada and Knight 141] then introduced the Phrase-based approach, which was subsequently improved by Koehn [142]. It translates based on phrases corresponding to more than one word. Thus, this approach is able to consider the surrounding context of each word when dealing with ambiguous words. The Phrase-based approach is considered a huge advancement with respect to MT approaches. However, problems such as reordering and ambiguity still exist especially when translating multi-domain contents, which basically do not have a vocabulary overlap. This lack of vocabulary is known as the out-of-vocabulary (OOV) problem, OOV words are unknown words which appear while testing a given MT approach but do not occur when training the model.

Regarding the remaining errors, one remedy found was to include linguistic rules in the translation model. Consequently, the Syntax-based (also known as Factored models) and Tree-based approaches appeared. They combine linguistics rules with statistical models, but the linguistic rules used by both approaches are different from those in RBMT. The difference lies in the limitations imposed by applying some complex linguistic rules in a polynomial efficient time. Thus, some crucial linguistic rules are not considered. The factored models use part-of-speech tagging, which is responsible for decreasing structural problems while Tree-based models attempt to resolve the reordering problem by using syntactic trees between languages. This combination solves basic and important problems caused by the structure of languages in Phrase-based MT. In spite of Tree-based approaches showing notable improvements, they skip a large number of necessary parsing rules, thus continuing to produce material errors [2].

Recently, a new statistical approach called Neural Networkbased (also known as NMT) ${ }^{19}$ has gained significant popularity [143, 144]. Although NN techniques are not essentially novel, they have been widely exploited since Google published their research [145] on beneficial improvements applying NN on sequence-to-sequence models ${ }^{20}$ The structure of a given NMT system is much simpler than the well-known Phrasebased SMT in terms of components. In NMT systems, there are no separate models (e.g., language model, translation model), rather, they usually consist of one sequence model responsible for encoding and decoding the words. This sequen-

${ }^{19}$ see new chapter at Koehn's book http://statmt.org/mtma16/ uploads/mtma16-neural.pdf

${ }^{20}$ http://www.statmt.org/survey/Topic/ NeuralNetworkModels 
ce model learns a context vector created from the sources and target sentences for translating the texts. The context vector, i.e., embeddings, is an important part as it represents the continuous space representation of words and allows the sequence model to predict similar words. Despite some researchers claiming that NMT may practically solve many of the weaknesses of SMT systems, it is still recent research and, as a statistical approach, still suffers from some well-known statistical problems [13] that are described in Section 3.3

\subsubsection{Example-based Machine Translation}

Similar to statistical approaches, EBMT use bilingual corpora, but they store the data by example sentences. The training phase of EBMT approaches imitates a basic memory technique (analogy) of human translators and is akin to filling the database of a translation memory system [146]. Here, EBMT approaches split the source text of a training corpus into sentences and align each of these sentences with the corresponding target sentences from the same training dataset. When an EBMT is used, the known source sentences are fetched first. If any of these sentences occur in the input, the machine replaces it by the corresponding translation. If during the translation an unknown word occurs in a sentence (i.e., a word which was not seen in the training data), the word is not translated. This is clearly the main limitation of EBMT approaches, which is addressed by hybrid MT systems. If several translations are available, the translation process chooses the target sentence based on a ranking algorithm. As EBMT systems are related to memory techniques, they require large physical memory to store the translated sentences. Although the MT community has moved forward with this approach, it is quite interesting and easily combined with the others.

\subsection{Problem space addressed by $M T$}

According to [136], some MT approaches may perform better than others for translating contents depending on the problem, language and how much bilingual content is available. Although RBMT approaches are still currently used due to the difficulty of finding bilingual or comparable corpora containing syntactic information for some morphologically rich languages, the MT community has practically moved on from RBMT approaches because of the complicated extension of their linguistic rules. It has occurred due to the exponential number of research works on SMT, based on languages which are widely spoken around the world and consequently have more bilingual data, such as English, French and Chinese. Thus, the creation of hand-crafted rules from these languages is no longer needed, as the ML algorithms seemed to resolve the problems.

Additionally, Koehn [2] states that acquiring comparable corpora is made simple by using crawling techniques on the Web. However, the process of alignment required by SMT approaches is substantially harder, and even more so without an explicit well-defined Web format. For instance, there is a task created by the well-known MT workshop with the goal of aligning sentences automatically from Web pages in order to create comparable corpor ${ }^{21}$ The results and the training phase of this task confirm the difficulty of the process, for example, the reasonable difference between possible and training pairs where the training pairs are 1,624 and possible pairs are 225,043. Possible pairs are pairs which exist from a human perspective and may be found by an algorithm. In contrast, training pairs are accurate pairs which are given for training the candidate algorithms. Although the results of this task are promising, the evaluated systems require improvements for creating a real MT training data. Also, most comparable corpora are limited in terms of domains (e.g politics, technology and financial).

\subsection{MT performance and effort}

All MT approaches have advantages and disadvantages w.r.t. quality and efforts. In Table 2, we compare the approaches presented in Section 3.2. considering the dimensions stated previously.

The main weakness of RBMT systems lies in their complexity of building the models, which is associated with the manual processing of deep linguistic knowledge. For example, Interlingual approaches require significant time to create the internal representation by linguists [148]. Linguistics professionals commonly need to annotate the rules and structure manually. Although RBMT are clearly rather expensive approaches, grammar problems may be found easily by linguists. Note that the modification of current rules does not guarantee better results. Linguists even may diverge from some rules, thus causing the creation of inconsistent rules. Consequently, the creation of accurate RBMT has come to be regarded as a very difficult task with many bottlenecks. Additionally, RBMT performs well with closely related languages, however, the performance drops between language pairs of significant difference.

On the other hand, SMT systems do not require complex linguistic rules. However, the main challenge is reaching a good translation quality over multi-domain texts. Commonly, SMT systems are trained using reference translations by which ML algorithms are able to analyze the data and find patterns by themselves, thus being able to translate text without any rules created by humans. Although some basic linguistics mistakes have been solved by Tree-based and Neural Networkbased approaches, the lack of complex linguistic rules still causes ambiguity problems (e.g., errors on relative pronouns)[149. An additional problem of the latter approach is the complexity of performing error analysis over outputs. For instance, NMT systems do not provide an easy way to find problems in a feasible time 150 151. In spite of the fact that NMT approaches have currently been achieving better results than the others, they have similar drawbacks and are much less interpretable than SMT. Additionally, NMT approaches struggles to deal with OOV words (rare words) since they have a fixed vocabulary size. However, the community has been combining efforts in order to handle this problem by using 
Table 2: MT Approaches Comparison

\begin{tabular}{|c|c|}
\hline \multicolumn{2}{|c|}{ Rule-based } \\
\hline Pros & Cons \\
\hline Deep linguistic knowledge (quality) $^{\mathrm{a}}$ & Requires linguistic rules and dictionaries (effort) $^{b}$ \\
\hline Easy to perform error analysis (effort) ${ }^{b}$ & Human language inconsistency (quality) $^{\mathrm{a}}$ \\
\hline & Expensive to extend and improve (effort) ${ }^{b}$ \\
\hline \multicolumn{2}{|r|}{ Statistical } \\
\hline Pros & Cons \\
\hline No complex processing of linguistic rules (effort) ${ }^{b}$ & Require parallel text ${ }^{\mathrm{c}}\left(\right.$ quality $^{\mathrm{a}}$, effort $^{\mathrm{b}}$ ) \\
\hline Less human resources cost, no linguists required (effort) ${ }^{b}$ & No linguistic rules causes syntactic/semantic errors (quality) ${ }^{a}$ \\
\hline Applicable to any pair of languages (effort) ${ }^{b}$ & Difficult to perform error analysis (effort) ${ }^{b}$, especially for NMT \\
\hline Models trained with human translations (quality) $^{\text {a }}$ & Preprocessing noisy training data (effort) ${ }^{b}$ \\
\hline
\end{tabular}

a Translation quality: In Pros, it means to improve the quality of outputs while it means to reduce the quality in Cons.

b Human efforts: In Pros, it means to decrease the human efforts while in Cons, it means to increases the human efforts.

c some NMT approaches have started to use only monolingual data for an unsupervised training [147.

character-based models or sub-words units, which are able to predict unforeseen words 152-154]. However, it is still an open problem which is related to the disambiguation of words. Furthermore, despite the advances in NMT approaches, a significant amount of effort is being invested in improving the scalability of RBMT approaches in order to achieve a higher performance than their counterparts. Hence, RBMT is still regarded as essential to high-quality translation, even more so for rich morphological languages.

\subsection{Open MT Challenges}

The most problematic unresolved MT challenges, from our point of view, which are still experienced by the aforementioned MT approaches are the following:

1. Complex semantic ambiguity: This challenge is mostly caused by the existence of homonymous and polysemous words. Given that a significant amount of parallel data is necessary to translate such words and expressions adequately. MT systems commonly struggle to translate these words correctly, even if the models are built upon from 5- or 7-grams. For example, "John promises to keep his room tidy" and "John has some promises to keep until he is trusted again". Although the meaning of both are clear to humans, these sentences for SMT systems are statistically expensive and prone to failure ${ }^{22}$ Additionally, for translating the simple word "bank", context information is essential for determining which meaning to assign to it.

2. Structural divergence: By definition, structural reordering is reorganizing the order of the syntactic constituents of a language according to its original structure [149]. It in turn becomes a critical issue because it is the core of the translation process. Every language has its own syntax, thus each MT system needs to have adequate models for the syntax of each language. For instance,

\footnotetext{
${ }^{22}$ See a complete discussion about the problem: http://tinyurl. com/yck5ngj8
}

reordering a sentence from Japanese to English is one of the most challenging techniques because of the SVO (subject-verb-object) and SOV (subject-object-verb) wordorder difference and also, one English word often groups multiple meanings of Japanese characters. For example, Japanese characters make subtle distinctions between homonyms that would not be clear in a phonetic language such as English.

3. Linguistic properties/features: A large number of languages display a complex tense system. When confronted with sentences from such languages, it can be hard for MT systems to recognize the current input tense and to translate the input sentence into the right tense in the target language. For instance, some irregular verbs in English like "set" and "put" cannot be determined to be in the present or past tense without previous knowledge or pre-processing techniques when translated to morphologically rich languages, e.g., Portuguese, German or Slavic languages. Additionally, the grammatical gender of words in such morphologically rich languages contributes to the problem of tense generation where a certain MT system has to decide which inflection to use for a given word. This challenge is a direct consequence of the structural reordering issue and remains a significant problem for modern translator systems.

Additionally, there are five MT open challenges posed by Lopez and Post [155] which we describe more generically below.

(1) Excessive focus on English and European languages as one of the involved languages in MT approaches and poor research on low-resource language pairs such as African and/or South American languages. (2) The limitations of SMT approaches for translating across domains. Most MT systems exhibit good performance on law and the legislative domains due to the large amount of data provided by the European Union. In contrast, translations performed on sports and life-hacks commonly fail, because of the lack of training data. (3) How to translate the huge amount of data from social networks that 
uniquely deal with no-standard speech texts from users (e.g., tweets). (4) The difficult translations among morphologically rich languages. This challenge shares the same problem with the first one, namely that most research work focuses on English as one of the involved languages. Therefore, MT systems which translate content between, for instance, Arabic and Spanish are rare. (5) For the speech translation task, the parallel data for training differs widely from real user speech.

The challenges above are clearly not independent, which means that addressing one of them can have an impact on the others. Since NMT has shown impressive results on reordering, the main problem turns out to be the disambiguation process (both syntactically and semantically) in SMT approaches [2].

\subsection{MT Evaluation Metrics}

An evaluation of a given MT system may be carried out either automatically or manually. Generally, the MT community has opted to use automatic metrics to decrease human efforts and time. Once an MT system has shown promising results from a certain automatic evaluation metric, the output of this MT system is then evaluated by a human translator. A common process of automatic evaluation is composed of the source text (input), target text (output produced by an MT system which is also called hypothesis) and the reference translation of the source text (output created by a human translator). The reference translation is compared automatically against the target text using a given evaluation metric. There are plenty of automatic MT evaluation metrics. However, below we introduce only the most important metrics which were also used for evaluating the surveyed papers. Such metrics enable a scientific comparison between the quality of different MT systems.

- BLEU was created by Papineni [156] as an attempt to decrease the professional translation efforts for evaluating the performance of MT systems. BLEU is widely chosen for evaluating MT outputs due to its low costs. BLEU uses a modified precision metric for comparing the MT output with the reference translation. The precision is calculated by measuring the ngram similarity (size 1-4) at word levels. BLEU also applies a brevity penalty by comparing the length of the MT output with the reference translation. Additionally, some BLEU variations have been proposed to improve its evaluation quality. The most common variation deals with the number variability (frequency) of useless words commonly generated by MT systems [157]. However, the main weakness of BLEU is its difficulty handling semantic variations (i.e., synonyms) while performing the ngram similarity.

- NIST was designed by Doddington [158 to address some weaknesses of BLEU, upon which it is based. Instead of attributing the same weight for each word in a sentence, NIST gives more weight to a rare word when matched by n-gram. It also modifies the penalty applied by
BLEU on the comparison of the MT output with the human translation, reducing the impact of small variations on the overall score.

- METEOR was introduced by Banerjee and Lavie [159 to overcome some weaknesses of BLEU and NIST, for example, the lack of explicit word-matching between translation and reference, the lack of recall and the use of geometric averaging of $n$-grams. The goal of METEOR is to use semantic features to improve correlation with human judgments of translation quality. To this end, METEOR considers the synonymy overlap through a shared WordNet synset of the words.

- TER is different from the aforementioned metrics. TER measures the number of necessary edits in an MT output to match the reference translation exactly. The goal of TER is to measure how much effort is needed to fix an automated translation to make it fluent and correct [160]. The edits consist of insertions, deletions, substitutions and shift of words, as well as capitalization and punctuation. The TER score is calculated by computing the number of edits divided by the average referenced words.

- MEANT was firstly created by Chi-kiu Lo in 2011 [161] to alleviate the semantic correlation deficit between the reference and MT outputs from well-known MT metrics such as METEOR and BLEU. Although METEOR has been created to deal with the semantic weakness of BLEU (i.e synonyms), it ignores the meaning structures of the translations. MEANT outperforms all the existing MT metrics in correlation with human adequacy judgment, and is relatively easy to port to other languages. MEANT requires only an automatic semantic parser and a monolingual corpus of the output language, which is used to train the discrete context vector model to compute the lexical similarity between the semantic role fillers of the reference and translation. Recently, a variation of MEANT has been created by Chi-kiu Lo et. al [162 which is currently the state-of-the-art of semantic MT evaluation metrics. This new version of MEANT replaced the discrete context vector model with continuous word embeddings in order to further improve the accuracy of MEANT. The accuracy of MEANT relies heavily on the accuracy of the model that determines the lexical similarities of the semantic role fillers.

- chrF proposed by Popović [163 164] was initially the use of character n-gram precision and recall (F-score) for automatic evaluation of MT outputs. Recently, Popović [165 enhanced chrF with word n-grams which has shown appealing results, especially for morphologically rich target languages. Although n-gram is already used in well-known and complex MT metrics, the investigation of n-grams as an individual metric has not been exploited before. chrF has shown a good correlation with human rankings of different MT outputs. chrF is simple 
and does not require any additional information. Additionally, chrF is language and tokenisation independent.

\section{Surveyed Papers}

In this section, we describe how the surveyed articles work and conclude with suggested directions for using SWT in $\mathrm{MT}^{23}$

\subsection{Selected Research Works}

This section describes all surveyed articles according to the MT approaches described in Section 3 in order to provide continuity for the reader. Table 3 presents a comparison of all surveyed articles, along with their MT method and applied SWT. The column MT approach represents which kind of MT was chosen for handling the translation process. The column SW method denotes what SWT were used to extract the knowledge contained in a given SW resource, for instance, semantic annotation technique, SPARQL queries, and reasoning. Semantic annotation is the process of inserting additional information or metadata to concepts in a given text or any other content. It enriches the content with machinereadable information. SPARQL is a Resource Description Framework (RDF) query language, which is a semantic query language for databases. Reasoning is the technique responsible for deriving unseen logical and sensible relations from a set of explicit facts or axioms. The column Resource shows what kind of resource was used to acquire the SW knowledge, in this case ontology files or Linked Open Data (LOD), which means a Knowledge Base (KB) such as DBpedia or BabelNet. The column Evaluation illustrates the evaluation process that was applied for measuring the quality of the work, e.g., whether the evaluation was performed by humans or using automatic evaluation metrics.

\subsubsection{Direct}

- Heuss et al.[124] combined SWT as a post-editing technique with the direct approach in their work. Post-editing technique involves fixing mistakes from a given output by choosing the right translated word or order. Although this technique is commonly used by a professional translator or a linguist, its automated implementation has been widely researched recently in order to reduce human efforts. The works [15, 124] propose a method for retrieving translations from a domain ontology. The approach performs SPARQL queries to search translations of a given word. The ontology uses SKOS vocabulary [166 to describe its multilingual content. SKOS is a common model for describing concepts in SW. It uses the prefLabel property to assign a primary label to a particular concept and altLabel for alternative names or translation $\$^{24}$ Once SPARQL queries have retrieved the

\footnotetext{
${ }^{23}$ The description of the articles may not follow a standard due to the lack of details in some works.

24 https: / / www.w3.org/TR/skos-reference/
}

translations, a reasoner infers a relationship between the source and target words based on its properties contained in the ontology. The authors manually marked parts of the data as triggers. Hence, the inference rules were basically done manually. Although this work provides important insights, the evaluation of its output is insubstantial as the authors evaluated only one sentence to validate their approach. The given explanation for this weak evaluation is that the reasoner is not fast enough for large texts and for executing on more than one sentence.

\subsubsection{Transfer-Based}

- In NERITS [125], the authors presented an MT system with additional information about the translated text. They used DBpedia to provide concepts about each word, similar to the semantic annotation technique. A semantic annotation technique involves annotating a given word or text, adding information/concepts about it from a given KB. The goal of NERITS is to provide more knowledge for users using SW. The authors chose a Transferbased approach for translating the content. The translation process begins performing Named Entity Recognition (NER) [167 through a tool called Wikimeta to deal with the variations of entities. The NER technique is responsible for recognizing the entities and their respective types. For instance, Microsoft is an organization and Bill Gates is a person. Thus, Wikimeta is responsible for linking each recognized entity with a given resource within the knowledge base. Although the authors contended for the use of a post-editing technique, the SW method applied here does not edit or improve the output, it just provides concepts for helping the user to understand and assimilate the translation. The authors stated that no current MT systems provide conceptual knowledge about the translated words, they only present the crude translation. Their goal was to describe how users can learn about languages combining MT with SW. The authors stated that their proposal could not be evaluated with automatic MT metrics because they did not improve the translation process, but annotated the translations providing additional knowledge.

- O. Lozynska and M. Davydov [128] developed an MT system for translation of Ukrainian Sign Language (USL). The authors chose the Transfer-based MT approach because of the lack of parallel data for statistical translation. Additionally, the authors argued that a rule-based approach, along with ontologies, is best suited for implementing the rules of sign languages. To this end, the authors used a grammatically augmented ontology from a variety of domains such as education, nature and army. The ontology was mainly used for supporting a given parser in the extraction of syntactic and semantic rules. These syntactic-semantic rules enable a deep analysis of sentences thus avoiding the problem 
Table 3: Details of surveyed articles

\begin{tabular}{|c|c|c|c|c|c|}
\hline Citation & Year & MT approach & SW method & SW resource & Evaluation \\
\hline C. Vertan 115 & 2004 & EBMT & Annotation & Ontologies & None \\
\hline N. Elita and A. Birladeanu 117 & 2005 & EBMT & SPARQL & Ontologies & None \\
\hline W. Hahn and C. Vertan [116] & 2005 & EBMT & SPARQL + Annotation & Ontologies & None \\
\hline C. Shi and H. Wang 118 & 2005 & None & Reasoner & Ontologies & None \\
\hline N. Elita and M. Gavrila 119 & 2006 & EBMT & SPARQL & Ontologies & Human \\
\hline E. Seo et al. 120 & 2009 & None & Reasoner & Ontologies & None \\
\hline P. Knoth et al. 121 & 2010 & RBMT or SMT & Annotation & Ontologies & Human \\
\hline L. Lesmo et al. [122] & 2011 & Interlingua & Annotation & Ontologies & Human \\
\hline \begin{tabular}{l|l|l|l|} 
B. Harriehausen-Mühlbauer and T. Heuss & 15 & 124 \\
\end{tabular} & 2012 & Direct & SPARQL + Reasoner & Ontologies & Human \\
\hline $\begin{array}{l}\text { K. Nebhi et al. } 125 \\
\end{array}$ & 2013 & TBMT & Annotation & LOD & None \\
\hline J. P. McCrae and P. Cimiano 126 & 2013 & SMT & Annotation & LOD & Human \\
\hline D. Moussallem and R. Choren 127 & 2015 & SMT & SPARQL & Ontologies & Human \\
\hline O. Lozynska and M. Davydov 128 & 2015 & RBMT & Annotation & Ontologies & Human \\
\hline K.Simov et al. 129 & 2016 & RBMT + SMT & SPARQL & LOD & Automatic \\
\hline N. Abdulaziz et al. [131 & 2016 & SMT & SPARQL & Ontologies & Human \\
\hline J. Du et al. 132 & 2016 & SMT & SPARQL & LOD & Automatic \\
\hline A. Srivastava et al. 133 & 2016 & SMT & SPARQL + Annotation & LOD & Automatic \\
\hline C. Shi et al. 134 & 2016 & NMT & Annotation & LOD & Automatic + Human \\
\hline A. Srivastava et al. 135 & 2017 & SMT & SPARQL + Annotation & LOD & Automatic \\
\hline
\end{tabular}

of ambiguity in USL. The crucial contribution according to authors was the creation of a new domain-specific language based on the ontologies which may be further used for editing and processing future works in the translation of USL using ontologies. The evaluation was carried out manually by linguists since the sign languages are difficult to be evaluated automatically. The results were quite promising, as the ontology contributed to $19 \%$ of improvements in the analysis of Ukrainian signs and $35 \%$ in the generation of Ukrainian natural language compared to baseline from related works.

\subsubsection{Interlingua}

- Lesmo et al. [122] presented an approach for translating from the Italian language to Italian Sign Language. The authors translated the content using the interlingual approach with SWT. The goal of using SWT, in this case an ontology, is to discern the syntactic ambiguity of a given word caused by the automatic syntaxsemantic interpretation within the generation step at the translation process. To this end, the authors used a very limited domain, a weather forecast ontology, for dealing with the variety of specific terms. Therefore, the authors assumed that a certain word meaning may be expressed by ontology nodes. Thus, the relationship between nodes which are ontology properties may resolve the syntax-semantic issues when translating a sentence. Afterwards, the authors converted the ontology structure into first-order logic and included the logic forms using the OpenCCG tool into the interlingual language model. Therefore, the translation starts by performing a syntactic analysis step which is based on dependency trees. Afterward, each word is annotated with its respective lexical meaning from the ontology, thus creating an ontological representation. This representation is then interpreted to determine which path (i.e., meaning) to follow and then translating the words correctly. The Interlingual approach was chosen because a translation of sign language requires deep linguistic expertise. Finally, the authors raised the problem of the lengthy time taken to translate due to the many ambiguity issues related to sign language and concluded that the approach requires further evaluation.

\subsubsection{Statistical}

- J. P. McCrae and P. Cimiano [126] extracted English-German translations from LOD. They used these results to improve the WSD inside SMT. The authors used the well-known SMT system called Moses [168], inserting the results retrieved from LOD into the phrase table created by Moses. The phrase table, which contains the frequency of the phrases, is used to select the best meaning (i.e., translation), thus discerning among various source meanings to arrive at a unique target meaning. Each word mined from LOD is queried in the Moses frequency table. If the word is not in the table, they insert the bestfit word (1.0) into the table. They did not achieve good results with automatic evaluation using BLEU [156]. The outcomes showed no improvement against Moses as a baseline system. Baseline achieved 11.80 while baseline+ $\mathrm{LD}$ achieved 11.78. However, the manual evaluation done by linguists showed a significant improvement of almost $50 \%$ percent in the output translation.

- Moussallem and Choren [127] presented a novel approach to tackle the ambiguity gap of message translations in dialogue systems. The translations occurred between Brazilian Portuguese and English. Currently, submitted messages to a dialogue system are considered as isolated sentences. Thus, missing context information im- 
pedes the disambiguation of homographs in ambiguous sentences. Their approach tries to solve this disambiguation problem by using concepts in different existing ontologies. First, the user log is parsed in order to find out the respective ontology that matches the dialogue context. Using the SKOS vocabulary, the ontology that returns the most results for each verb or noun is used as context. When the SMT system returns a translation indicating a homonymous word (i.e., many different possible translations), this method queries the word in the ontology using SPARQL and replaces the target word accordingly. The authors evaluated their approach manually using empirical methods. The ontologies were Music ontology ${ }^{25}$ and Vehicle ontology ${ }^{26}$. Regarding its focus on dialogue systems, the work fails to translate slang or expressions that are common in dialogues. Since such sentences are rarely in a wellstructured form, the translations often contain structural errors. Also, the context is fixed during a dialogue, so a topic change is not reflected by the system ${ }^{27}$

- Neama Abdulaziz et al. [131 extended the previously described approach presented by Moussallem et al. to Arabic-English translations. As its main extension, this work includes a dependency tree parser because of the rich morphological structure of Arabic. Thus, the SMT approach used by authors is Tree-based, which inserts the tree as statistical rules. In contrast, during the common training phase of SMT, the dependency rules are attached according to the sent message. The authors use the MS-ATKS tool for analyzing the syntactic structure of messages and also a domain ontology on the Quran. Once this work has been applied to dialogue systems, the evaluation is carried out empirically by humans. The authors propose to apply different automatic metrics as further plans in order to evaluate additional aspects of this work.

- Santosh Kumar T.S. [130] proposed an approach to address lexical disambiguation in the translation of proper nouns from English to Dravidian languages such as Tamil. To this end, the authors created a corpus containing only ambiguous sentences for testing their approach. They used Google Translate as an SMT system because, in accordance with the state-of-the-art, Google MT system has the best translation model for translating from English to Tamil. The approach preprocessed ambiguous sentences using an NER tool for recognizing persons. Additionally, they relied on FOAF ontology in RDF structure for supporting the translation. This ontology contains information about a large number of names in Tamil. Due to the lack of resources in the Tamil language, which are required for automatic metrics such

25 http://musicontology.com/

26 http://www.heppnetz.de/ontologies/vso/ns

27 https://github.com/DiegoMoussallem/judgemethod as BLEU, the evaluation was carried out manually. It presented positive outcomes where the professional translator confirmed the capability of SWT to support translations of entities among morphologically rich languages.

- Jinhua Du et al. [132 created an approach addressing the problem of OOV words. These kinds of words do not appear in the translation table of a given SMT approach such as Phrase-based. Commonly, they are named entities which often appear on the Web, such as persons and organizations, but they can also be common words like "kiwi" which is highly ambiguous. Therefore, the authors proposed three methods to deal with the OOV words problem using a SW resource called BabelNet. The respective methods are: (1) Direct Training, which retrieves every pair of source and target words, creating a dictionary to use during the training phase. (2) Domain-Adaption, which recognizes the subject of source corpus and applies a topic-modelling technique as Moussallem et al. have done. Hence, the found subject adapts the target corpus for a specific domain, gathering the information from BabelNet. According to the authors, these first two methods did not perform well, so they decided to apply BabelNet as (3) Post-processing technique. To this end, they used an SMT decoder in order to recognize OOV words which were not translated beforehand. Subsequently, they performed SPARQL queries through BabelNet API [169] to retrieve translations of these words. As in previously surveyed works, they used Moses as a baseline for an SMT system to perform experiments. Additionally, they chose Chinese, English and Polish languages for translating the contents and decided to evaluate the translations from their system using the automatic metrics BLEU and TER. The evaluation showed unstable results using BabelNet. However, it also uncovered a promising way to rectify the OOV problem by looking for unknown words in LD. The authors intend to investigate different forms of using SWT in MT systems as a future work.

- A. Srivastava et al. [133 implemented a novel framework which translates named entities in SMT systems using LOD resources. The framework is akin to the approach of J. P. McCrae and P. Cimiano [126] and it uses Moses as an SMT baseline system. Natural Language Processing Interchange Format (NIF) vocabulary is used as interchanging format for converting natural language sentences to SW structure (i.e., triples). Then, the authors used DBpedia Spotlight [170] to recognize entities, thus facilitating their translation. Once the entities are recognized, their respective translations are gathered using SPARQL through the DBpedia endpoint. The translation of entities is marked using XML format and inserted into a Moses translation table. These markings avoid Moses having to translate the entities using its own probabilistic model and forces Moses to select the translations retrieved from LOD resources. The evaluation was carried out using the IT domain data from 
the MT workshop ${ }^{28}$ which consists of 1.000 sentence translations. This task uses BLEU and TER for measuring the performance of a given MT system. Their LDbased framework showed a significant improvement of $12 \%$ in comparison to the Moses baseline. BLEU increased by $0.8 \%$ and TER decreased by $3.2 \%$. The authors dubbed their framework 'SW-aware MT system'. Although they provided links to projects related to this framework, there are no links to their implementation.

- C. Shi et al [134 built a semantic embedding model relying on knowledge-bases to be used in NMT systems. The work is dubbed Knowledge Base Semantic Embedding (KBSE), which consists of mapping a source sentence to triples and then using these triples to extract the internal meaning of words to generate a target sentence. The mapping results in a semantic embedding model containing KB triples which were responsible for gathering the key information of each word in the sentences. Therefore, the authors investigated the contribution of KB to enhance the quality of the translation of Chinese-English MT systems. To this end, they applied KBSE in two domain-specific datasets, electric business, and movies. The evaluation was two-fold. First, they compared KBSE with a standard NMT system and also with Moses as SMT system by using BLEU as an automatic evaluation metric. Second, they selected humans to manually evaluate the translations. Additionally, the authors used an external named entity translator to get the translation of English entities into Chinese. Moreover, they included this entity translator in the evaluation of the other MT systems for a fair comparison. The results of KBSE were quite promising, BLEU showed an improvement of 1.9 points (electric) and 3.6 (movie) when compared to the standard encoder-decoder NMT system. Additionally, using the KBSE method received much higher results than using the Moses toolkit. This work shows that a given neural model, when trained using semantic information gathered from a KB, is able to memorize the key information of source sentences. Also, their model rarely made grammatical mistakes as the authors expected because of the strong learning ability of Gated Recurrent Unit [171]. Moreover, KBSE presented some errors when translating entities due to the external entity translator. Furthermore, since KBSE comprises two separate models, when an error appears at the source part, it can not be corrected in the target part.

- A. Srivastava et al. 135 mixed approaches from their last work [133] with some strategies implemented by Jinhua Du et al. 132 and the approach implemented by J. P. McCrae and P. Cimiano [126]. This work therefore comprises of three strategies for using $\mathrm{LD}$ with Moses as an SMT system. In the first strategy, the authors used the LD resources as dictionaries for word alignment. Thus the translation models contained knowledge from the LD resources and also from bilingual corpora. Hence, the Moses decoder is able to decide which translation of a given word to choose. This strategy is akin to J. P. McCrae and P. Cimiano's [126] work. The second strategy relied on their former work, [133] which forced the Moses decoder to retrieve the translation of a given named entity by performing Entity Linking (EL) along with SPARQL queries from LD. The last strategy was inspired by Jinhua Du et al.'s [132] work. It applied a post-editing technique to correct the OOV words, which means untranslated words in MT output. The authors evaluated their approach using BabelNet, DBpedia and JRC names as LOD resources on the WMT12 datase ${ }^{29}$ The evaluation showed only modest improvements by BLEU and TER metrics because both deal poorly with semantics, i.e, synonyms. Therefore, the real contribution of LD in this work was made clear when the evaluation was performed by humans.

\subsubsection{Example-Based}

EBMT was the first methodology proposed to work alongside SWT because of its architecture. The EBMT process is simpler than other methods, thus facilitating its combination with other technologies. All works discussed in this section were carried out by a single research group. However, the research was discontinued.

- Vertan [115] presented an architecture based on EBMT which retrieves word meanings using semantic annotation from ontologies. As SW was in its infancy in 2004 when this work was undertaken, it proposed an architecture but did not implement it or give details properly. Thus, it is unclear how the semantic annotation actually helped the translation process.

- Her second work with Hahn [116 showed how semantic annotations could be used in MT systems. They explained how the properties in RDF data structures could support the annotation of words in several languages. Vertan and Hahn presented the same architecture of Vertan's previous work [115], but did not show the process of evaluation.

- A year later, Elita et al. [117 proposed a prototype of EBMT where SW was more concrete, which queried sentences from ontologies. The sentences were extracted from the text and queried through SPARQL endpoints instead of common databases. The translation was only provided by the ontology, which retrieved exactly one translation for each source sentence. Consequently, this work showed how Vertan's architecture could be implemented [115]. However, no evaluation was provided. 
- Subsequently, N. Elita and M. Gavrila [119] used an ontology to map existing concepts in a source text using NER and summarization techniques. The authors used five languages to prove their method. This approach showed how an ontology can support an automatic WSD process in MT. The authors stated that there was no need to analyze the text syntactically. However, this lack of analysis prevented the approach from handling inflections properly, which is a requirement for achieving good translations and thus a negative point of this work. The evaluation was done manually and demonstrated solid improvements only on translation templates, which their approach identified beforehand, but failed to present a comparison to other example-based approaches.

\subsubsection{Hybrid}

Whilst the combination of multiple MT approaches is already a challenge, three works were found that integrate a hybrid approach with SWT.

- Almasoud et al. 123 mix the translation process of TBMT (a rule-based approach) with EBMT (a corpus-based one) while using a domain ontology. This work translates from the Arabic language to Arabic Sign language. According to the authors, TBMT was chosen because a sign language requires deep linguistic knowledge (like 122 did in subsubsection 4.1.3. First, the text is converted into a representation model based on Arabic sign language rules, then the model is translated into signals. The ontology which belongs to the religion domain is applied in a similar fashion to the WSD task, exploiting its semantic knowledge. Then, every word is searched in the ontology to find its respective sign. In the case where there is no sign for a certain word, the SignWriting corpus is used to retrieve respective synonyms. Like many other works in this survey, this work fails to provide a detailed evaluation. The authors argued that an evaluation with automatic metrics is not possible because these do not provide measures to evaluate sign languages. Thus, they performed a manual evaluation, but did not provide any measure of improvement.

- Knoth et al. [121] presented an approach combining CrossLanguage Information Retrieval (CLIR) and MT approaches to gather translations of specific terminologies from domain ontologies. CLIR is a technique for retrieving contents from languages other than the language used for searching. They did not mention which kind of RBMT and SMT method they combined. Their approach is divided into two phases: (1) The initialization phase is responsible for creating the monolingual ontology and generating the lightweight multilingual ontology using a given RBMT system. To do this they first collected the monolingual text from the European Government and built a simple ontology using the SKOS vocabulary. Using RBMT, they translated the monolingual terms into nine European languages, thus creating a multilingual lightweight ontology. This multilingual ontology is then evaluated by domain experts, concluding the first step. (2) The bootstrapping phase then applied CLIR techniques by querying terms through SPARQL. Multilingual terms were used to create parallel corpora for each of the nine languages in order to train the SMT. Regarding the parallel corpora, they extracted translation pairs and updated the terms when a new document was submitted. Their approach allows the user to decide which domain and language to use. The authors emphasized that the CLIR method can be adopted by any MT system. Furthermore, they stressed that multilingual ontologies support translations between any pair of languages. They also did not perform a proper evaluation of their architecture.

- Simov et al. [129] aimed to create a semantic-based hybrid MT system between Bulgarian and English in the domain of information technology. Their system supports the automatic identification of appropriate answers to user questions in a multilingual question/answering system. The authors chose RBMT because Bulgarian is a morphologically rich language. However, they do not mention which rule-based approach was used. Along with the RBMT, they used dictionaries and a PoS tagger in order to create rules which were then included in the SMT model akin to a tree-based approach. Consequently, the SMT system is the main part of their MT system. They used a parallel corpus as a gold standard dataset from EUROPARL ${ }^{30}$ to evaluate their approach. Additionally, they used two WSD techniques which were supported by OntoWordNet and LT4eL [172]. Although they used LT4eL as a domain ontology, they suggested that DBpedia may be used to address other domains, because DBpedia fails in covering the information technology domain. LT4el was included in OntoWordNet which is their previous work [173]. Thus, they were able to support WSD through the WordNet synsets inside Moses SMT system. The evaluation, comparing their hybrid MT system with the Moses system as baseline, showed no improvement for the BLEU metric. However, when the authors evaluated using the NIST metric, their system showed real improvement. The authors propose to generate more sophisticated rules for further improvements.

\subsubsection{New perspective-Ontology-based MT}

In this section, we describe two articles using SWT to translate contents without relying on well-known MT approaches. They present an MT system mainly based on SW methods.

- In the first work, Seo et al. [120] use an ontology created by themselves from a gold standard corpus by performing the ontology learning method [174]. This ontology is responsible for providing semantic knowledge 
of words. The syntactic analysis uses formal language expressed using Extended Backus-Naur Form (EBNF) notation [175] to match the English patterns defined beforehand. These patterns are only defined in the English language, hence the MT system only translates contents from English to Korean. Their system is not able to perform a translation in both directions (round-trip translations). Co-occurring words in the corpus are represented by relationship properties in the ontology.

Thus, the algorithm performs WSD by analyzing the ontological relationships between part-of-speech tags for deciding the best translation. For instance, the Korean language is well-known to group meaning of several words together, like the Chinese language. Thus, when translating a given word, its meaning has to be determined. In the example they gave, they attributed the translation of "hard" to a given Korean character according to the part-of-speech given by the EBNF notation. Then, they were able to search for a translation of "hard" as an adverb having the meaning "with effort". This method is comparable to a bag-of-words algorithm, but with additional graph features provided by the ontology to deal with Korean language structure.

- The second work by Shi et al. [118 proposes an ontology they also created. The ontology "SCIENTIST" describes terms in the physical science domain and was derived from other ontologies like SUMO. It is used to improve the semantic quality in their Chinese-English MT. The syntactic analysis part is done using Lexical Functional Grammar [176]. For the semantic analysis, their WSD is achieved by comparing relationship weighting between the parts-of-speech of the words in the ontology, similar to the previous work.

Both articles show promising approaches. Even though these works are quite similar, the second is much more detailed and its concepts are more applicable in future works and for supporting other languages. Moreover, unlike the first, the second work is capable of making a round translation. Neither work includes an evaluation, and comparing their results directly is impossible due to their different domain ontologies. Consequently, they are not capable of translating content across contexts. Unfortunately, their source codes are also not publicly available.

\subsection{Suggestions and Possible Directions}

According to the surveyed papers, SWT have mostly been applied at the semantic analysis step, rather than at the other stages of the translation process, due to their ability to deal with concepts behind the words and provide knowledge about them. Table 4 presents an overview of the surveyed papers, regarding each generic step of the MT approaches supported by SWT.

As SWT have developed, they have increasingly been able to resolve some of the open challenges of MT described in Section 3.5 They may be applied in different ways according to each MT approach. Although the potential graph structure contained in ontologies may act as a disambiguation method with high decision power, some SW concepts, such as the alignment of multilingual ontologies and the linking among monolingual knowledge bases, [177, 178] still need to be improved before SWT can be applied successfully in MT systems.

Additionally, the SW community has worked out basic suggestions for generating structured data. However, there are no well-defined rules for building the KBs, only common best practices according to Zaveri et al. [179]. Due to this lack of defined standards, some research works have produced erroneous ontologies or knowledge graphs into LOD repositories. Common issues include the following mistakes:

- Lack of well-defined object properties, e.g., cardinality or reflexiveness.

- Mixing of concepts among thesaurus, vocabulary, and ontology.

- Incorrect domain and range definitions.

- Use of ambiguous annotations.

Therefore, applying SWT to MT may seem difficult. However, recently some efforts from the Linguistic Linked Open Data (LLOD) ${ }^{31}$ community have been directed to modeling linguistic phenomena, linguistic rules and translations in LOD [180].

In the following subsections, we discuss and suggest future potential solutions to MT challenges by applying SWT. From our point of view, SWT mostly contribute to addressing syntactic and semantic ambiguity problems in MT systems. Thus, we divide our suggestions into four categories according to their respective problem.

\subsubsection{Disambiguation}

Human language is very ambiguous. Most words have multiple interpretations depending on the context in which they are mentioned. In the MT field, WSD techniques are concerned with finding the respective meaning and correct translation to these ambiguous words in target languages. This ambiguity problem was identified early in MT development. In 1960 Bar-Hillel [4] stated that an MT system is not able to find the right meaning without a specific knowledge. Although the ambiguity problem has been lessened significantly since the contribution of Carpuat and subsequent works [181[183], this problem still remains a challenge. As seen in Section 3 . MT systems still try to resolve this problem by using domain specific language models to prefer domain specific expressions, but when translating a highly ambiguous sentence or a short text which covers multiple domains, the languages models are not enough.

According to surveyed articles, SW has already shown its capability for semantic disambiguation of polysemous and homonymous words. However, SWT were applied in two ways

\footnotetext{
$3 1 \longdiv { \text { http://linguistics.okfn.org/ } }$
} 
Table 4: Semantic Web technologies in Machine Translation steps

\begin{tabular}{|c|c|c|c|c|c|c|}
\hline \multirow{3}{*}{ MT approaches } & \multicolumn{6}{|c|}{ MT Architecture } \\
\hline & \multicolumn{3}{|c|}{ Analysis } & \multicolumn{3}{|c|}{ Generation } \\
\hline & Morphological & Syntactic & Semantic & Morphological & Syntactic & Semantic \\
\hline Direct & $\mathrm{X}$ & & & $\mathrm{X}$ & & \\
\hline Transfer-based & & $\mathrm{X}$ & & & $\mathrm{X}$ & $\mathrm{X}$ \\
\hline Interlingua & & $\mathrm{X}$ & $\mathrm{X}$ & & $\mathrm{X}$ & $\mathrm{X}$ \\
\hline Statistical & & & $\mathrm{X}$ & & & $\mathrm{X}$ \\
\hline Example-based & $\mathrm{X}$ & & $\mathrm{X}$ & & & $\mathrm{X}$ \\
\hline Hybrid & $\mathrm{X}$ & $\mathrm{X}$ & $\mathrm{X}$ & $\mathrm{X}$ & & \\
\hline
\end{tabular}

to support the semantic disambiguation in MT. First, the ambiguous words were recognized in the source text before carrying out the translation, applying a pre-editing technique. Second, SWT were applied to the output translation in the target language as a post-editing technique. Although applying one of these techniques has increased the quality of a translation, both techniques are tedious to implement when they have to translate common words instead of named entities, then be applied several times to achieve a successful translation. The real benefit of SW comes from its capacity to provide unseen knowledge about emergent data, which appears every day. Therefore, we suggest performing the topicmodelling technique over the source text to provide a necessary context before translation. Instead of applying the topicmodeling over the entire text, we would follow the principle of communication (i.e from 3 to 5 sentences for describing an idea [184]) and define a context for each piece of text. Thus, at the execution of a translation model in a given SMT, we would focus on every word which may be a homonymous or polysemous word. For every word which has more than one translation, a SPARQL query would be required to find the best combination in the current context. Thus, at the translation table, the disambiguation algorithm could search for an appropriate word using different SW resources, such as BabelNet [185] or DBpedia, in consideration of the context provided by the topic modelling. The goal is to exploit the use of more than one SW resource at once for improving the translation of ambiguous terms. The use of two or more SW resources simultaneously has not yet been investigated.

On the other hand, there is also a syntactic disambiguation problem which as yet lacks good solutions. For instance, the English language contains irregular verbs like "set" or "put". Depending on the structure of a sentence, it is not possible to recognize their verbal tense, e.g., present or past tense. Even statistical approaches trained on huge corpora may fail to find the exact meaning of some words due to the structure of the language. Although this challenge has successfully been dealt with since NMT has been used for European languages [186, implementations of NMT for some non-European languages have not been fully exploited (e.g., Brazilian Portuguese, LatinAmerica Spanish, Hindi) due to the lack of large bilingual data sets on the Web to be trained on. Thus, we suggest gathering relationships among properties within an ontology by using the reasoning technique for handling this issue. For instance, the sentence "Anna usually put her notebook on the table for studying" may be annotated using a certain vocabulary and represented by triples. Thus, the verb "put", which is represented by a predicate that groups essential information about the verbal tense, may support the generation step of a given MT system. This sentence usually fails when translated to rich morphological languages, such as Brazilian-Portuguese and Arabic, for which the verb influences the translation of "usually" to the past tense. In this case, a reasoning technique may support the problem of finding a certain rule behind relationships between source and target texts in the alignment phase (training phase).

Reasoning techniques have been identified as a possible future way of supporting MT tasks because some remaining MT issues cannot be solved with explicit knowledge only. For instance, in accordance with Manning [187], some syntactic ambiguity gaps need to be addressed by human knowledge. This kind of human knowledge can be gained using reasoning over ontological relations described by humans. For example, in 2004 Legrand and Pulido [188 combined ontological knowledge with neural algorithms to perform the WSD task. The result was very promising, but unfortunately the work was discontinued. Additionally, some researchers, including Harriehausen-Mühlbauer and Heuss and Seo et al. [120, 124], have used reasoners to disambiguate words in MT systems.

For a given reasoning technique to be required, some previous steps need to be addressed. Currently, the OntologyLexica Community Grour ${ }^{32}$ at W3C has combined efforts to represent lexical entries, with their linguistic information, in ontologies across languages. Modeling different languages using the same model may provide an alignment between the languages, where it is possible to infer new rules using the language dependency graph structure and visualize a similarity among languages. ${ }^{33}$ SW tools that perform reasoning and infer unseen concepts are called Reasoners. Examples include Pellet, RACER, FACT++, and DL-Learner [189]. For the sake of clarification, DL-Learner [190] would learn concepts about a specific source or target text modeled by Ontolex. Thus, DL-Learner could infer new facts across related languages.

\footnotetext{
32 https://www.w3.org/community/ontolex/

${ }^{33}$ This insight is already supported by a recent publication in Cicling conference https://www.cicling.org/2017/posters.html named "The Fix-point of Dependency Graph - A Case Study of Chinese-German Similarity" by Tiansi Dong et al.
} 
Moreover, there are plenty of other ontologies and vocabularies which may support the description of languages, such as Olia [191], Lemon [192], GOLD [193] and NIF [194]. However, the aforementioned ontology issues may limit reasoner in its ability to support translations. Besides this drawback, a well-known problem of reasoners is the poor run-time performance. Therefore, they are not suitable for real-time MT approaches at this point in time. Furthermore, both deficiencies need to be addressed or minimized before implementing reasoners successfully into MT systems.

\subsubsection{Named Entities}

Most Named Entity Recognition and Disambiguation (NERD) approaches link recognized entities with database entries or websites [167]. This method helps to categorize and summarize text, but also contributes to the disambiguation of words in texts. The primary issue in MT systems is caused by common words from a source language that are used as proper nouns in a target language. For instance, the word "Kiwi" is a family name in New Zealand which comes from the Māori culture, but it also can be a fruit, a bird, or a computer program. Named Entities are a common and difficult problem in both MT (see Koehn [2]) and SW fields. The SW achieved important advances in NERD using structured data and semantic annotations, e.g., by adding an $r d f: t y p e$ statement which identifies whether a certain kiwi is a fruit [195, 196]. In MT systems, however, this problem is directly related to the ambiguity problem and therefore has to be resolved in that wider context.

Although MT systems include good recognition methods, NERD techniques still need improvement. When an MT system does not recognize an entity, the translation output often has poor quality, immediately deteriorating the target text readability. Therefore, we suggest recognizing such entities before the translation process and first linking them to a reference knowledge base. Afterwards, the type of entities would be agglutinated along with their labels and their translations from a reference knowledge base. For instance, in NMT, the idea is to include in the training set for the aforementioned word "Kiwi", "Kiwi.animal.link, Kiwi.person.link, Kiwi.food.link" then finally to align them with the translations in the target text. In SMT, the additional information would be included by Extensible Markup Language (XML) or by an additional model. This method would also contribute to OOV mistakes regarding names. This idea is akin to [134] where the authors encoded the types of entities along with the words to improve the translation of sentences between Chinese-English.

Another potential solution could apply type extraction or co-occurrence techniques and combine them with NERD methods. The solution would identify which pronouns (e.g., "it") are related to entities that have already been mentioned in previous sentences. This would also improve the fluency of target texts. In addition to our suggestions, the contextual linking over texts can help users to acquire a deeper understanding of the translated content (as NERITS has done). Plenty of SW tools would be able to support this approach in accordance with Gangemi [197. This work provides a complete comparison of SW tools, detailing their powerful capabilities for extracting knowledge.

\subsubsection{Non-standard speech}

The non-standard language problem is a rather important one in the MT field. Many people use the colloquial form to speak and write to each other on social networks. Thus, when MT systems are applied on this context, the input text frequently contains slang, Multiword Expressions (MWE), and unreasonable abbreviations such as "Idr = I don't remember." and "cya $=$ see you". Additionally, idioms contribute to this problem, decreasing the translation quality. Idioms often have an entirely different meaning than their separated word meanings. Consequently, most translation outputs of such expressions contain errors.

For a good translation, the MT system needs to recognize such slang and try to map it to the target language. Some SMT systems like Google or Bing have recognition patterns over non-standard speech from old translations through the Web using SMT approaches. In rare cases SMT can solve this problem, but considering that new idiomatic expressions appear every day and most of them are isolated sentences, this challenge still remains open. Moreover, each person has their own speaking form.

Therefore, we suggest that user characteristics can be applied as context for solving the non-standard language problem. These characteristics can be extracted from social media or user logs and stored as user properties using SWT, e.g., FOAF or SIOC [198] vocabularies. These ontologies have properties which would help identify the birth place or the interests of a given user. For instance, the properties foaf:interest and sioc:topic can be used to describe a given person's topics of interest. If the person is a computer scientist and the model contains topics such as "Information Technology" and "Sports", the SPARQL queries would search for terms inserted in this context which are ambiguous. Furthermore, the property foaf:based_near may support the problem of idioms. Assuming that a user is located in a certain part of Russia and he is reading an English web page which contains some idioms, this property may be used to gather appropriate translations of idioms from English to Russian using a given RDF KB [199. Therefore, an MT system can be adapted to a user by using specific data about him in RDF along with given KBs.

\subsection{Translating KBs}

According to the surveyed articles, it is clear that SWT may be used for translating KBs in order to be applied in MT systems. For instance, some content provided by the German Wikipedia version are not contained in the Portuguese one. Therefore, the semantic structure (i.e., triples) provided by DBpedia versions of these respective Wikipedia versions would be able to help translate from German to Portuguese. For example, the terms contained in triples would be translated to a given target language using a dictionary containing domain words. This dictionary may be acquired in two different ways. First, by performing localisation, as in the works by J. P. 
McCrae 200] and Arcan [201, 202 which translates the terms contained in a monolingual ontology, thus generating a bilingual ontology. Second, by creating embeddings of both DBpedia versions in order to determine the similarity between entities through their vectors. This insight is supported by some recent works, such as Ristoski et al. [203], which creates bilingual embeddings using RDF based on Word2vec [204] algorithms, and Muhao Chen et al. [205], which generates multilingual knowledge graph embeddings for aligning entities across languages. Therefore, we suggest investigating an MT approach mainly based on SWT using NN for translating KBs. Once the KBs are translated, we suggest including them in the language models for improving the translation of entities.

Besides C. Shi et al [134], one of the recent attempts in this direction was carried out by Arčan and Buitelaar [206] ${ }^{34}$ The authors aimed to translate domain-specific expressions represented by English KBs in order to make the knowledge accessible for other languages. They claimed that KBs are mostly in English, therefore they cannot contribute to the problem of MT to other languages. Thus, they translated two KBs belonging to medical and financial domains, along with the English Wikipedia, to German. Once translated, the KBs were used as external resources in the translation of German-English. For the sake of brevity, they evaluated their approach in Phrasebased and NMT systems. The results were quite appealing and the further research into this area should be undertaken.

\section{Conclusions and Future Work}

In this paper, we detailed a systematic literature review of MT using SWT for translating natural language sentences. The review aimed to answer the four research questions defined in Section 2 by a thorough analysis of the 21 most relevant papers.

Our goal was to provide a clear understanding of how SWT have helped the translation process within MT systems. Few studies have been found, suggesting that this method is still in its infancy. The surveyed articles demonstrate that SWT have been mainly used for the disambiguation task in MT systems and their capabilities have steadily increased. Considering the decision power of SWT, they cannot be ignored by future MT systems.

Nevertheless, there are still significant drawbacks. Although the research shows strong evidence of general SW advantages in the translation process, as measured by automatic evaluation metrics, the real semantic contributions were assessed manually and the evaluators measured improvement according to the respective domains through which the work was approached. BLEU was the automatic evaluation metric used by all research works and it lacks semantic measurement, thus diminishing the real contribution of SW in MT. Recent works on MT evaluation have shown promising advances for dealing with semantics in related metrics. These recent works [207.

\footnotetext{
${ }^{34}$ This paper was not included in this survey, because it was not peerreviewed yet.
}

208 attached SWT, in this case DBnary [209], to METEOR to handle meanings for non-English languages. In addition, MEANT has become the state-of-the-art in terms of evaluating semantics correlations in translated texts. Therefore, we expect that upcoming research works attempting to combine SWT with MT will be better evaluated by using these methods.

How can SWT enhance MT quality? Regarding this main research question, we identified that both semantic and syntactic disambiguation, including entities, structural divergence and OOV words problems can all be tackled with SWT. Additionally, SWT and MT approaches face the problem of languages being inherently ambiguous, in terms of lexicon, syntax, semantics and pragmatics. Identifying concepts in a $\mathrm{KB}$ or finding the right translation for a word are instances of the same WSD problem. Therefore, a deeper understanding of how pieces of information obtained from ontologies and KBs on the one hand, and parallel and monolingual corpora on the other hand, may contribute solving these ambiguities in MT. The two most recently surveyed articles, [134. [135], which have implemented a translation model relying on structured knowledge, support our conclusion. They showed promising results even using an automatic evaluation metric, which does not consider semantics. Therefore, this work increases the evidence that SWT can significantly enhance the quality of MT systems.

Furthermore, we answered the first sub-question RQ1 by listing and discussing all surveyed articles. RQ2 is summarized in Table 3 where we list all applied SW methods and respective SW resources. For RQ3, in Section 4 we also discussed the impact of applying an ontology to MT. We conclude that ontological knowledge is generally beneficial for certain translation quality issues, mostly related to disambiguation in morphologically rich languages and sign languages. The ontological knowledge was a crucial resource for improving the translation between sign and spoken natural languages. We sought to answer RQ4, about the availability of SW-tools, and we could perceive that most of them were discontinued. Only AGDISTIS [195, 210], DBpedia Spotlight[170] and Babelfy [211], which had supported some of the surveyed articles, are currently available. Moreover, we noticed that these SW tools performed only a specific task - disambiguation, for supporting the translations. Apart from the tools, BabelNet and DBpedia were used as KBs by almost of all surveyed works.

As a next step, we intend to elaborate a novel MT approach based on our suggestions made in Section 4 . This involves an approach capable of simultaneously gathering knowledge from different SW resources for addressing the ambiguity of named entities, which can also alleviate the problem of OOV words. This insight relies on recent works [212, 213], which have guided the usage of external knowledge in NMT systems for overcoming the vocabulary limitation of NN models. Also, we aim to create a method to structure natural language sentences into triples for supporting the generation task.

Future works that can be expected from fellow researchers, based on statements in their own papers, include the creation of linguistic ontologies describing the syntax of rich morpho- 
logically languages for supporting MT approaches. In addition, alignment between ontologies is expected to try to bridge gaps that are not addressed by the current SMT models. Since well-known bilingual dictionaries have been mapped to RDF, the creation of multilingual dictionaries has become easier for content translation. These RDF dictionaries can help to improve MT steps, such as alignment, or even translate, based entirely on such semantic resources.

\section{Acknowledgements}

This research has been partially supported by the Brazilian National Council for Scientific and Technological Development (CNPq) (no. 206971/2014-1) and the H2020 HOBBIT Project (GA No. 688227)

\section{References}

[1] J. Slocum, A survey of machine translation: its history, current status, and future prospects, Computational linguistics 11 (1) (1985) 1-17.

[2] P. Koehn, Statistical Machine Translation, Cambridge University Press, 2010.

[3] D. Jurafsky, Speech and language processing: An introduction to natural language processing, Prentice Hall, 2000.

[4] Y. Bar-Hillel, The present status of automatic translation of languages, in: Advances in computers, Vol. 1, Elsevier, 1960, pp. 91-163.

[5] W. J. Hutchins, H. L. Somers, An introduction to machine translation, Vol. 362, Academic Press London, 1992.

[6] M. Popović, Class error rates for evaluation of machine translation output, in: Proceedings of the Seventh Workshop on Statistical Machine Translation, Association for Computational Linguistics, 2012, pp. 71-75.

[7] M. R. Costa-Jussa, M. Farrús, J. B. Mariño, J. A. Fonollosa, Study and comparison of rule-based and statistical Catalan-Spanish machine translation systems, Computing and Informatics 31 (2) (2012) 245270.

[8] G. Thurmair, Comparing rule-based and statistical MT output, in: The Workshop Programme, 2004, p. 5.

[9] P. F. Brown, J. Cocke, S. A. D. Pietra, V. J. D. Pietra, F. Jelinek, J. D. Lafferty, R. L. Mercer, P. S. Roossin, A statistical approach to machine translation, Computational linguistics 16 (2) (1990) 79-85.

[10] M. R. Costa-Jussa, J. A. Fonollosa, Latest trends in hybrid machine translation and its applications, Computer Speech \& Language 32 (1) (2015) 3-10.

[11] M. R. Costa-jussà, How much hybridization does machine translation Need?, Journal of the Association for Information Science and Technology 66 (10) (2015) 2160-2165.

[12] G. Thurmair, Comparing different architectures of hybrid Machine Translation systems, MT Summit XII: proceedings of the twelfth Machine Translation Summit (2009) 340-347.

[13] P. Koehn, R. Knowles, Six Challenges for Neural Machine Translation, arXiv preprint arXiv:1706.03872.

[14] T. Berners-Lee, J. Hendler, O. Lassila, The Semantic Web, Scientific american 284 (5) (2001) 34-43.

[15] T. Heuss, Lessons learned (and questions raised) from an interdisciplinary Machine Translation approach, in: Position paper for the W3C Workshop on the Open Data on the Web, 2013, pp. 23-24.

[16] T. Dyba, T. Dingsoyr, G. K. Hanssen, Applying Systematic Reviews to Diverse Study Types: An Experience Report, in: Proceedings of the First International Symposium on Empirical Software Engineering and Measurement, ESEM '07, IEEE Computer Society, Washington, DC, USA, 2007, pp. 225-234.

[17] B. Kitchenham, Procedures for performing systematic reviews, Keele, UK, Keele University 33 (2004) (2004) 1-26.

[18] D. Moher, A. Liberati, J. Tetzlaff, D. G. Altman, P. Group, et al., Preferred reporting items for systematic reviews and meta-analyses: the PRISMA statement, PLoS medicine 6 (7) (2009) el000097.
[19] A. Ettinger, S. Gella, M. Labeau, C. O. Alm, M. Carpuat, M. Dredze (Eds.), Proceedings of the 55th Annual Meeting of the Association for Computational Linguistics, ACL, Vancouver, Canada, 2017.

[20] K. Erk, N. A. Smith (Eds.), Proceedings of the 54th Annual Meeting of the Association for Computational Linguistics, ACL, Berlin, Germany, 2016.

[21] C. Zong, M. Strube (Eds.), Proceedings of the 53rd Annual Meeting of the Association for Computational Linguistics and the 7th International Joint Conference on Natural Language Processing, ACL, Beijing, China, 2015.

[22] K. Toutanova, H. Wu (Eds.), Proceedings of the 52nd Annual Meeting of the Association for Computational Linguistics, ACL, Baltimore, Maryland, 2014.

[23] T. H. K. U. o. S. Pascale Fung, Technology, U. o. E. Massimo Poesio (Eds.), Proceedings of the 51st Annual Meeting of the Association for Computational Linguistics (Volume 1: Long Papers), ACL, Sofia, Bulgaria, 2013.

[24] H. Li, C.-Y. Lin, M. Osborne, G. G. Lee, J. C. Park (Eds.), Proceedings of the 50th Annual Meeting of the Association for Computational Linguistics (Volume 1: Long Papers), ACL, Jeju Island, Korea, 2012.

[25] Y. Matsumoto, R. Mihalcea (Eds.), Proceedings of the 49th Annual Meeting of the Association for Computational Linguistics: Human Language Technologies, ACL, Portland, Oregon, USA, 2011.

[26] J. Hajič, S. Carberry, S. Clark, J. Nivre (Eds.), Proceedings of the 48th Annual Meeting of the Association for Computational Linguistics, ACL, Uppsala, Sweden, 2010.

[27] K.-Y. Su, J. Su, J. Wiebe, H. Li (Eds.), Proceedings of the Joint Conference of the 47th Annual Meeting of the ACL and the 4th International Joint Conference on Natural Language Processing of the AFNLP, ACL, Suntec, Singapore, 2009.

[28] J. D. Moore, S. Teufel, J. Allan, S. Furui (Eds.), Proceedings of the 46th Annual Meeting of the Association of Computational Linguistics, ACL, Columbus, Ohio, 2008.

[29] A. Zaenen, A. van den Bosch (Eds.), Proceedings of the 45th Annual Meeting of the Association of Computational Linguistics, ACL, Prague, Czech Republic, 2007.

[30] N. Calzolari, C. Cardie, P. Isabelle (Eds.), Proceedings of the 21st International Conference on Computational Linguistics and 44th Annual Meeting of the Association for Computational Linguistics, ACL, Sydney, Australia, 2006.

[31] K. Knight, H. T. Ng, K. Oflazer (Eds.), Proceedings of the 43rd Annual Meeting of the Association for Computational Linguistics, ACL, Ann Arbor, Michigan, 2005.

[32] D. Scott, W. Daelemans, M. A. Walker (Eds.), Proceedings of the 42nd Annual Meeting of the Association for Computational Linguistics, ACL, Barcelona, Spain, 2004.

[33] E. W. Hinrichs, D. Roth (Eds.), Proceedings of the 41st Annual Meeting of the Association for Computational Linguistics, ACL, Sapporo, Japan, 2003.

[34] E. W. Hinrichs, D. Roth (Eds.), Proceedings of the 40th Annual Meeting of the Association for Computational Linguistics, ACL, Philadelphia, PA, USA, 2002.

[35] K. Knight, A. Nenkova, O. Rambow (Eds.), Proceedings of the 2016 Conference of the North American Chapter of the Association for Computational Linguistics: Human Language Technologies, ACL, San Diego, California, 2016.

[36] R. Mihalcea, J. Y. Chai, A. Sarkar (Eds.), Proceedings of the 2015 Conference of the North American Chapter of the Association for Computational Linguistics: Human Language Technologies, ACL, Denver, Colorado, USA, 2015.

[37] L. Vanderwende, H. D. III, K. Kirchhoff (Eds.), Proceedings of the 2013 Conference of the North American Chapter of the Association of Computational Linguistics: Human Language Technologies, ACL, Atlanta, Georgia, USA, 2013.

[38] E. Fosler-Lussier, E. Riloff, S. Bangalore (Eds.), Proceedings of the 2012 Conference of the North American Chapter of the Association for Computational Linguistics: Human Language Technologies, ACL, Montréal, Canada, 2012.

[39] R. Kaplan, J. Burstein, M. Harper, G. Penn (Eds.), Proceedings of the 2010 Annual Conference of the North American Chapter of the Association for Computational Linguistics: Human Language Technologies, 
ACL, Los Angeles, California, 2010.

[40] M. Ostendorf, M. Collins, S. Narayanan, D. W. Oard, L. Vanderwende (Eds.), Proceedings of the 2009 Annual Conference of the North American Chapter of the Association for Computational Linguistics: Human Language Technologies, ACL, Boulder, Colorado, 2009.

[41] C. Sidner, T. Schultz, M. Stone, C. Zhai (Eds.), Proceedings of 2007 Annual Conference of the North American Chapter of the Association for Computational Linguistics: Human Language Technologies, ACL, Rochester, New York, 2007.

[42] R. C. Moore, J. Bilmes, J. Chu-Carroll, M. Sanderson (Eds.), Proceedings of 2006 Annual Conference of the North American Chapter of the Association for Computational Linguistics: Human Language Technologies, ACL, New York City, USA, 2006.

[43] R. Mooney, C. Brew, P. C.-c. Lee-Feng Chien, Academia Sinica, P. C.-c. Katrin Kirchhoff, University of Washington (Eds.), Proceedings of 2005 Annual Conference of the North American Chapter of the Association for Computational Linguistics: Human Language Technologies, ACL, Vancouver, British Columbia, Canada, 2005.

[44] H. T. Ng, E. Riloff (Eds.), Proceedings of 2004 Annual Conference of the North American Chapter of the Association for Computational Linguistics: Human Language Technologies, ACL, Boston, Massachusetts, USA, 2004.

[45] W. Daelemans, M. Osborne (Eds.), Proceedings of 2003 Annual Conference of the North American Chapter of the Association for Computational Linguistics: Human Language Technologies, ACL, Edmonton, Canada, 2003.

[46] M. Palmer, R. Hwa, S. Riedel (Eds.), Proceedings of the 2017 Conference on Empirical Methods in Natural Language Processing, ACL, Copenhagen, Denmark, 2017.

[47] J. Su, X. Carreras, K. Duh (Eds.), Proceedings of the 2016 Conference on Empirical Methods in Natural Language Processing, ACL, Texas, USA, 2016.

[48] L. M. rquez, C. Callison-Burch, J. Su (Eds.), Proceedings of the 2015 Conference on Empirical Methods in Natural Language Processing, ACL, Lisbon, Portugal, 2015.

[49] Q. C. R. I. Alessandro Moschitti, G. Bo Pang, U. o. A. Walter Daelemans (Eds.), Proceedings of the 2014 Conference on Empirical Methods in Natural Language Processing, ACL, Doha, Qatar, 2014.

[50] D. Yarowsky, T. Baldwin, A. Korhonen, K. Livescu, S. Bethard (Eds.), Proceedings of the 2013 Conference on Empirical Methods in Natural Language Processing, ACL, Seattle, Washington, USA, 2013.

[51] J. Tsujii, J. Henderson, M. PaÃě̈̌Äca (Eds.), Proceedings of the 2012 Joint Conference on Empirical Methods in Natural Language Processing and Computational Natural Language Learning, ACL, Jeju Island, Korea, 2012.

[52] R. Barzilay, M. Johnson (Eds.), Proceedings of the 2011 Conference on Empirical Methods in Natural Language Processing, ACL, Edinburgh, Scotland, UK., 2011.

[53] H. Li, L. M'arquez (Eds.), Proceedings of the 2010 Conference on Empirical Methods in Natural Language Processing, ACL, Cambridge, MA, 2010.

[54] P. Koehn, R. Mihalcea (Eds.), Proceedings of the 2009 Conference on Empirical Methods in Natural Language Processing, ACL, Singapore, 2009.

[55] M. Lapata, H. T. Ng (Eds.), Proceedings of the 2008 Conference on Empirical Methods in Natural Language Processing, ACL, Honolulu, Hawaii, 2008.

[56] J. Eisner (Ed.), Proceedings of the 2007 Joint Conference on Empirical Methods in Natural Language Processing and Computational Natural Language Learning (EMNLP-CoNLL), ACL, Prague, Czech Republic, 2007.

[57] D. Jurafsky, É. Gaussier (Eds.), Proceedings of the 2007 Conference on Empirical Methods in Natural Language Processing, ACL, Sydney, Australia, 2006.

[58] R. Mooney, C. Brew, P. C.-c. Lee-Feng Chien, Academia Sinica, P. C.-c. Katrin Kirchhoff, University of Washington (Eds.), Proceedings of the 2005 Conference on Empirical Methods in Natural Language Processing, ACL, Vancouver, British Columbia, Canada, 2005.

[59] R. Mooney, C. Brew, A. S. Lee-Feng Chien (Eds.), Proceedings of the 2004 Conference on Empirical Methods in Natural Language Processing, ACL, Barcelona Spain, 2004.
[60] N. Calzolari, Y. Matsumoto, R. Prasad (Eds.), Proceedings of COLING, the 26th International Conference on Computational Linguistics, ACL, Osaka, Japan, 2014

[61] J. Tsujii, J. Hajic (Eds.), Proceedings of COLING, the 25th International Conference on Computational Linguistics, ACL, Dublin, Ireland, 2014.

[62] M. Kay, C. Boitet (Eds.), Proceedings of COLING, the 24th International Conference on Computational Linguistics, ACL, Mumbai, India, 2012.

[63] C.-R. Huang, D. Jurafsky (Eds.), Proceedings of COLING, the 23th International Conference on Computational Linguistics, ACL, Beijing, China, 2010.

[64] D. Scott, H. Uszkoreit (Eds.), Proceedings of COLING, the 22th International Conference on Computational Linguistics, ACL, Manchester, UK, 2008.

[65] N. Calzolari, C. Cardie, P. Isabelle (Eds.), Proceedings of COLING, the 21sd International Conference on Computational Linguistics, ACL, Sydney, Australia, 2006.

[66] N. Calzolari, C. Cardie, P. Isabelle (Eds.), Proceedings of COLING, the 20th International Conference on Computational Linguistics, ACL, Geneva, Switzerland, 2004

[67] N. Calzolari, C. Cardie, P. Isabelle (Eds.), Proceedings of COLING, the 19th International Conference on Computational Linguistics, ACL, Taipei, Taiwan, 2002.

[68] R. Barrett, R. Cummings, E. Agichtein, E. Gabrilovich (Eds.), WWW '17 Proceedings of the 26th International Conference on World Wide Web, ACM, Perth, Australia, 2017.

[69] J. Bourdeau, J. Hendler, R. Nkambou, I. Horrocks, B. Y. Zhao (Eds.), WWW' 16 Proceedings of the 25th International Conference on World Wide Web, ACM, Montreal, Canada, 2016.

[70] S. Srinivasan, K. Ramamritham, A. Kumar, M. P. Ravindra, E. Bertino, R. Kumar (Eds.), WWW'11: Proceedings of the 20th International Conference on World Wide Web, ACM, New York, NY, USA, 2011.

[71] A. Mille, F. L. Gandon, J. Misselis, M. Rabinovich, S. Staab (Eds.), WWW '12: Proceedings of the 21st International Conference on World Wide Web, ACM, New York, NY, USA, 2012.

[72] D. Schwabe, V. A. F. Almeida, H. Glaser, R. A. Baeza-Yates, S. B. Moon (Eds.), WWW' 13 Companion: Proceedings of the 22Nd International Conference on World Wide Web Companion, International World Wide Web Conferences Steering Committee, Rio de Janeiro, Brazil, 2013.

[73] C. Chung, A. Z. Broder, K. Shim, T. Suel (Eds.), WWW' 14: Proceedings of the 23rd International Conference on World Wide Web, ACM, New York, NY, USA, 2014.

[74] A. Gangemi, S. Leonardi, A. Panconesi (Eds.), WWW' 15 Companion: Proceedings of the 24th International Conference on World Wide Web Companion, ACM, Republic and Canton of Geneva, Switzerland, 2015.

[75] P. Buitelaar, P. Cimiano, E. Montiel-Ponsoda (Eds.), Proceedings of the 1st International Workshop on the Multilingual Semantic Web, CEURWS.org, Raleigh, North Carolina, USA, 2010.

[76] C. Bizer, T. Heath, T. Berners-Lee, K. Idehen (Eds.), Proceedings of the WWW2009 Workshop on Linked Data on the Web, LDOW, CEURWS.org, Madrid, Spain, 2009.

[77] C. Bizer, T. Heath, K. Idehen, T. Berners-Lee (Eds.), Proceedings of the WWW2008 Workshop on Linked Data on the Web, LDOW, CEURWS.org, Beijing, China, 2008.

[78] P. Bouquet, H. Stoermer, G. Tummarello, H. Halpin (Eds.), Proceedings of the WWW2007 Workshop I ${ }^{3}$ : Identity, Identifiers, Identification, Entity-Centric Approaches to Information and Knowledge Management on the Web, CEUR-WS.org, Banff, Canada, 2007.

[79] L. Carr, D. D. Roure, A. Iyengar, C. A. Goble, M. Dahlin (Eds.), WWW '06: Proceedings of the 15th International Conference on World Wide Web, ACM, Edinburgh, Scotland, UK, 2006.

[80] A. Ellis, T. Hagino (Eds.), WWW' 05 : Proceedings of the 14th International Conference on World Wide Web, ACM, Chiba, Japan, 2005.

[81] S. I. Feldman, M. Uretsky, M. Najork, C. E. Wills (Eds.), WWW '04: Proceedings of the 13th International Conference on World Wide Web, ACM, New York, NY, USA, 2004.

[82] G. Hencsey, B. White, Y. R. Chen, L. Kovács, S. Lawrence (Eds.), WWW '03: Proceedings of the Twelfth International Conference on World Wide Web, ACM, Budapest, Hungary, 2003.

[83] D. Lassner, D. D. Roure, A. Iyengar (Eds.), WWW'02: Proceedings of the 
Eleventh International Conference on World Wide Web, ACM, Honolulu, Hawaii, 2002.

[84] V. Y. Shen, N. Saito, M. R. Lyu, M. E. Zurko (Eds.), WWW '01: Proceedings of the tenth International Conference on World Wide Web, ACM, Hong Kong, China, 2001.

[85] I. Horrocks, J. A. Hendler (Eds.), The Semantic Web - ISWC 2002, Springer, Sardinia, Italy, 2002.

[86] D. Fensel, K. P. Sycara, J. Mylopoulos (Eds.), The Semantic Web - ISWC 2003, Springer, Sanibel Island, FL, USA, 2003.

[87] S. A. McIlraith, D. Plexousakis, F. van Harmelen (Eds.), The Semantic Web - ISWC 2004, Springer, Hiroshima, Japan, 2004.

[88] Y. Gil, E. Motta, V. R. Benjamins, M. A. Musen (Eds.), The Semantic Web - ISWC 2005, Springer, Galway, Ireland, 2005.

[89] I. F. Cruz, S. Decker, D. Allemang, C. Preist, D. Schwabe, P. Mika, M. Uschold, L. Aroyo (Eds.), The Semantic Web - ISWC 2006, Vol. 4273, Springer, Athens, GA, USA, 2006.

[90] K. Aberer, K. Choi, N. F. Noy, D. Allemang, K. Lee, L. J. B. Nixon, J. Golbeck, P. Mika, D. Maynard, R. Mizoguchi, G. Schreiber, P. CudréMauroux (Eds.), The Semantic Web - ISWC 2007, Vol. 4825, Springer, Busan, Korea, 2007.

[91] A. P. Sheth, S. Staab, M. Dean, M. Paolucci, D. Maynard, T. W. Finin, K. Thirunarayan (Eds.), The Semantic Web - ISWC 2008, Springer, Karlsruhe, Germany, 2008.

[92] A. Bernstein, D. R. Karger, T. Heath, L. Feigenbaum, D. Maynard, E. Motta, K. Thirunarayan (Eds.), The Semantic Web - ISWC 2009, Springer, Chantilly, VA, USA, 2009.

[93] P. F. Patel-Schneider, Y. Pan, P. Hitzler, P. Mika, L. Zhang, J. Z. Pan, I. Horrocks, B. Glimm (Eds.), The Semantic Web-ISWC 2010, SpringerVerlag, Berlin Heidelberg, Germany, 2010.

[94] L. Aroyo, H. Welty, Chris Alani, J. Taylor, A. Bernstein, L. Kagal, N. Noy, E. Blomqvist (Eds.), The Semantic Web-ISWC 2011, Springer-Verlag, Berlin Heidelberg, Germany, 2011.

[95] P. Cudré-Mauroux, J. Heflin, E. Sirin, T. Tudorache, J. Euzenat, M. Hauswirth, J. X. Parreira, J. Hendler, G. Schreiber, A. Bernstein, E. Blomqvist (Eds.), The Semantic Web-ISWC 2012, Springer-Verlag, Berlin Heidelberg, Germany, 2012.

[96] H. Alani, L. Kagal, A. Fokoue, P. Groth, C. Biemann, J. X. Parreira, L. Aroyo, N. Noy, C. Welty, K. Janowicz (Eds.), The Semantic Web-ISWC 2013, Springer-Verlag, Berlin Heidelberg, Germany, 2013.

[97] P. Mika, T. Tudorache, A. Bernstein, C. Welty, C. A. Knoblock, D. Vrandecic, P. T. Groth, N. F. Noy, K. Janowicz, C. A. Goble (Eds.), The Semantic Web - ISWC 2014, Riva del Garda, Italy, October 19-23, 2014. Proceedings, Vol. 8796, Springer, 2014.

[98] M. Arenas, Ó. Corcho, E. Simperl, M. Strohmaier, M. d'Aquin, K. Srinivas, P. T. Groth, M. Dumontier, J. Heflin, K. Thirunarayan, S. Staab (Eds.), The Semantic Web - ISWC 2015, Springer, Bethlehem, PA, USA, 2015.

[99] P. T. Groth, E. Simperl, A. J. G. Gray, M. Sabou, M. Krötzsch, F. Lécué, F. Flöck, Y. Gil (Eds.), The Semantic Web - ISWC 2016 - 15th International Semantic Web Conference, Springer, Kobe, Japan, 2016.

[100] C. d'Amato, M. Fernández, V. A. M. Tamma, F. Lécué, P. CudréMauroux, J. F. Sequeda, C. Lange, J. Heflin (Eds.), The Semantic Web - ISWC 2017 - 16th International Semantic Web Conference, Lecture Notes in Computer Science, Springer, Vienna, Austria, 2017.

[101] E. Blomqvist, D. Maynard, A. Gangemi, R. Hoekstra, P. Hitzler, O. Hartig (Eds.), The Semantic Web - 14th International Conference, ESWC, Lecture Notes in Computer Science, Portorož, Slovenia, 2017.

[102] H. Sack, E. Blomqvist, M. d'Aquin, C. Ghidini, S. P. Ponzetto, C. Lange (Eds.), The Semantic Web. Latest Advances and New Domains, Vol. 9678 of Lecture Notes in Computer Science, Springer, Berlin Heidelberg, Germany, 2016.

[103] G. Antoniou, M. Grobelnik, E. Simperl, B. Parsia, D. Plexousakis, P. de Leenheer, J. Z. Pan (Eds.), The Semantic Web: Research and applications, Vol. 6643 of Lecture Notes in Computer Science, Semantic Technology Institute International (STI2), Springer-Verlag, Berlin Heidelberg, Germany, 2011.

[104] E. Simperl, P. Cimiano, A. Polleres, O. Corcho, V. Presutti (Eds.), The Semantic Web: Research and applications, Vol. 7295 of Lecture Notes in Computer Science, Semantic Technology Institute International (STI2), Springer-Verlag, Berlin Heidelberg, Germany, 2012.

[105] P. Cimiano, O. Corcho, V. Presutti, L. Hollink, S. Rudolph (Eds.), The
Semantic Web: Semantics and Big Data, Vol. 7882 of Lecture Notes in Computer Science, Semantic Technology Institute International (STI2), Springer-Verlag, Berlin Heidelberg, Germany, 2013.

[106] V. Presutti, C. d'Amato, F. Gandon, M. d'Aquin, S. Staab, A. Tordai (Eds.), The Semantic Web: Trends and Challenges, Vol. 8465 of Lecture Notes in Computer Science, Semantic Technology Institute International (STI2), Springer International Publishing, Cham, Switzerland, 2014.

[107] F. Gandon, M. Sabou, H. Sack, P., A. Zimmermann (Eds.), The Semantic Web. Latest Advances and New Domains, Vol. 9088 of Lecture Notes in Computer Science, Semantic Technology Institute International (STI2), Springer International Publishing, Cham, Switzerland, 2015.

[108] L. Aroyo, G. Antoniou, E. Hyvönen, A. ten Teije, H. Stuckenschmidt, L. Cabral, T. Tudorache (Eds.), The Semantic Web: Research and Applications, 7th Extended Semantic Web Conference, ESWC 2010, Springer, Heraklion, Crete, Greece, 2010.

[109] L. Aroyo, P. Traverso, F. Ciravegna, P. Cimiano, T. Heath, E. Hyvönen, R. Mizoguchi, E. Oren, M. Sabou, E. P. B. Simperl (Eds.), The Semantic Web: Research and Applications, 6th European Semantic Web Conference, ESWC 2009, Springer, Heraklion, Crete, Greece, 2009.

[110] S. Bechhofer, M. Hauswirth, J. Hoffmann, M. Koubarakis (Eds.), The Semantic Web: Research and Applications, 5th European Semantic Web Conference, ESWC 2008, Springer, Tenerife, Canary Islands, Spain, 2008.

[111] E. Franconi, M. Kifer, W. May (Eds.), The Semantic Web: Research and Applications, 4th European Semantic Web Conference, ESWC 2007, Springer, Innsbruck, Austria, 2007.

[112] Y. Sure, J. Domingue (Eds.), The Semantic Web: Research and Applications, 3rd European Semantic Web Conference, ESWC 2006, Springer, Budva, Montenegro, 2006.

[113] A. Gómez-Pérez, J. Euzenat (Eds.), The Semantic Web: Research and Applications, Second European Semantic Web Conference, ESWC 2005, Springer, Crete, Greece, 2005.

[114] C. Bussler, J. Davies, D. Fensel, R. Studer (Eds.), The Semantic Web: Research and Applications, First European Semantic Web Symposium, ESWS, Springer, Crete, Greece, 2004

[115] C. Vertan, Language resources for the Semantic Web: perspectives for machine translation, in: Proceedings of the Second International Workshop on Language Resources for Translation Work, Research and Training, ACL, 2004, pp. 37-41.

[116] W. Hahn, C. Vertan, Challenges for the Multilingual Semantic Web, in: Proceedings of the International MT Summit X, 2005, pp. 5-9.

[117] N. Elita, A. Birladeanu, A first step in integrating an EBMT into the Semantic Web, in: Proceedings of the International MT Summit X, Phuket, Thailand, 2005, pp. 1-6.

[118] C. Shi, H. Wang, Research on ontology-driven Chinese-English machine translation, in: Natural Language Processing and Knowledge Engineering, 2005. IEEE NLP-KE'05. Proceedings of 2005 IEEE International Conference on, IEEE, 2005, pp. 426-430.

[119] N. Elita, M. Gavrila, Enhancing translation memories with semantic knowledge, in: Proceedings of the First Central European Student Conference in Linguistics, 2006, pp. 24-26.

[120] E. Seo, I.-S. Song, S.-K. Kim, H.-J. Choi, Syntactic and semantic English-Korean machine translation using ontology, in: Advanced Communication Technology, 2009. ICACT 2009. 11th International Conference on, Vol. 3, IEEE, 2009, pp. 2129-2132.

[121] P. Knoth, T. Collins, E. Sklavounou, Z. Zdrahal, Facilitating crosslanguage retrieval and machine translation by multilingual domain ontologies, in: Workshop on Supporting eLearning with Language Resources and Semantic Data, Citeseer, 2010, p. 51.

[122] L. Lesmo, A. Mazzei, D. P. Radicioni, An ontology based architecture for translation, in: Proceedings of the Ninth International Conference on Computational Semantics, Association for Computational Linguistics, 2011, pp. 345-349.

[123] A. M. Almasoud, H. S. Al-Khalifa, A proposed semantic machine translation system for translating Arabic text to Arabic sign language, in: Proceedings of the Second Kuwait Conference on e-Services and eSystems, ACM, 2011, p. 23.

[124] B. Harriehausen-Mühlbauer, T. Heuss, Semantic web based machine translation, in: Proceedings of the Joint Workshop on Exploiting 
Synergies between Information Retrieval and Machine Translation (ESIRMT) and Hybrid Approaches to Machine Translation (HyTra), Association for Computational Linguistics, 2012, pp. 1-9.

[125] K. Nebhi, L. Nerima, E. Wehrli, NERITS-A Machine Translation Mashup System Using Wikimeta and DBpedia, in: The Semantic Web: ESWC 2013 Satellite Events, Springer, 2013, pp. 312-318.

[126] J. P. McCrae, P. Cimiano, Mining translations from the web of open linked data, in: Joint Workshop on NLP\&LOD and SWAIE: Semantic Web, Linked Open Data and Information Extraction, 2013, p. 8.

[127] D. Moussallem, R. Choren, Using Ontology-Based Context in the Portuguese-English Translation of Homographs in Textual Dialogues, Artificial Intelligence and Applications 1510.

[128] O. Lozynska, M. Davydov, Information technology for Ukrainian Sign Language translation based on ontologies, ECONTECHMOD: an international quarterly journal on economics of technology and modelling processes 4 (2) (2015) 13-18.

[129] K. Simov, P. Osenova, A. Popov, Towards Semantic-based Hybrid Machine Translation between Bulgarian and English, Proceedings of the 2016 Conference of the North American Chapter of the Association for Computational Linguistics: Human Language Technologies.

[130] T. Santosh Kumar, Word Sense Disambiguation Using Semantic Web for Tamil to English Statistical Machine Translation, IRA-International Journal of Technology \& Engineering 5 (2) (2016) 22-31.

[131] I. A. A.-B. G. H. G. Neama Abdulaziz Bin-Dahan, Fadl Mutaher Ba-Alwi, Towards an Arabic-English Machine-Translation Based on Semantic Web, in: SCITN, Vol. 1, 2016, p. 6.

[132] J. Du, A. Way, A. Zydron, Using babelnet to improve OOV coverage in SMT, in: Proceedings of the Tenth International Conference on Language Resources and Evaluation LREC 2016, Portorož, Slovenia, May 23-28, 2016., 2016, pp. 9-15.

[133] A. Srivastava, F. Sasaki, P. Bourgonje, J. M. Schneider, J. Nehring, G. Rehm, How to Configure Statistical Machine Translation with Linked Open Data Resources, in: Translating and the Computer 38 Proceedings, AsLing, 2016, pp. 138-148.

[134] C. Shi, S. Liu, S. Ren, S. Feng, M. Li, M. Zhou, X. Sun, H. Wang, Knowledge-Based Semantic Embedding for Machine Translation, in: Proceedings of the 54th Annual Meeting of the Association for Computational Linguistics (Volume 1: Long Papers), Vol. 1, 2016, pp. 22452254.

[135] A. Srivastava, G. Rehm, F. Sasaki., Improving Machine Translation through Linked Data, in: Improving Machine Translation through Linked Data., The Prague Bulletin of Mathematical Linguistics - EAMT, 2017, pp. 355-366.

[136] P. Bhattacharyya, Machine Translation, Chapman and Hall/CRC, 2015.

[137] P. F. Brown, V. J. D. Pietra, S. A. D. Pietra, R. L. Mercer, The mathematics of statistical machine translation: Parameter estimation, Computational linguistics 19 (2) (1993) 263-311.

[138] P. Li, A survey of machine translation methods, Indonesian Journal of Electrical Engineering and Computer Science 11 (12) (2013) 71257130.

[139] M. A. Chéragui, Theoretical overview of machine translation, Proceedings ICWIT (2012) 160.

[140] A. Ranta, Grammatical framework: Programming with multilingual grammars, CSLI Publications, Center for the Study of Language and Information, 2011.

[141] K. Yamada, K. Knight, A syntax-based statistical translation model, in: Proceedings of the 39th Annual Meeting on Association for Computational Linguistics, Association for Computational Linguistics, 2001, pp. 523-530.

[142] P. Koehn, F. J. Och, D. Marcu, Statistical phrase-based translation, in: Proceedings of the 2003 Conference of the North American Chapter of the Association for Computational Linguistics on Human Language Technology-Volume 1, Association for Computational Linguistics, 2003, pp. 48-54.

[143] N. Kalchbrenner, P. Blunsom, Recurrent Continuous Translation Models., in: EMNLP, Vol. 3, 2013, p. 413.

[144] D. Bahdanau, K. Cho, Y. Bengio, Neural machine translation by jointly learning to align and translate, arXiv preprint arXiv:1409.0473.

[145] I. Sutskever, O. Vinyals, Q. V. Le, Sequence to sequence learning with neural networks, in: Advances in neural information processing systems, 2014, pp. 3104-3112.
[146] D. D. King, R. J. Redpath, Method and system for improving machine translation accuracy using translation memory, uS Patent 6,278,969 (August 2001).

[147] G. Lample, L. Denoyer, M. Ranzato, Unsupervised machine translation using monolingual corpora only, CoRR abs/1711.00043.

[148] B. J. Dorr, E. H. Hovy, L. S. Levin, Machine translation: Interlingual methods, Natural Language Processing and Machine Translation Encyclopedia of Language and Linguistics, 2nd ed. (ELL2).

[149] A. Bisazza, M. Federico, A survey of word reordering in statistical machine translation: Computational models and language phenomena, Computational Linguistics.

[150] M. Johnson, M. Schuster, Q. V. Le, M. Krikun, Y. Wu, Z. Chen, N. Thorat, F. Viégas, M. Wattenberg, G. Corrado, et al., Google's Multilingual Neural Machine Translation System: Enabling Zero-Shot Translation, arXiv preprint arXiv:1611.04558.

[151] Y. Wu, M. Schuster, Z. Chen, Q. V. Le, M. Norouzi, W. Macherey, M. Krikun, Y. Cao, Q. Gao, K. Macherey, et al., Google's Neural Machine Translation System: Bridging the Gap between Human and Machine Translation, arXiv preprint arXiv:1609.08144.

[152] R. Sennrich, B. Haddow, A. Birch, Neural machine translation of rare words with subword units, in: Proceedings of the 54th Annual Meeting of the Association for Computational Linguistics (Volume 1: Long Papers), Association for Computational Linguistics, 2016, pp. 1715-1725.

[153] M.-T. Luong, C. D. Manning, Achieving open vocabulary neural machine translation with hybrid word-character models, in: Proceedings of the 54th Annual Meeting of the Association for Computational Linguistics (Volume 1: Long Papers), Association for Computational Linguistics, 2016, pp. 1054-1063.

[154] J. Chung, K. Cho, Y. Bengio, A character-level decoder without explicit segmentation for neural machine translation, in: Proceedings of the 54th Annual Meeting of the Association for Computational Linguistics (Volume 1: Long Papers), Association for Computational Linguistics, 2016, pp. 1693-1703.

[155] A. Lopez, M. Post, Beyond bitext: Five open problems in machine translation, in: Proceedings of the EMNLP Workshop on Twenty Years of Bitext, 2013, pp. 1-3.

[156] K. Papineni, S. Roukos, T. Ward, W.-J. Zhu, BLEU: a method for automatic evaluation of machine translation, in: Proceedings of the 40th annual meeting on association for computational linguistics, Association for Computational Linguistics, 2002, pp. 311-318.

[157] B. Babych, A. Hartley, Extending the bleu mt evaluation method with frequency weightings, in: Proceedings of the 42nd annual meeting on association for computational linguistics, Association for Computational Linguistics, 2004, p. 621.

[158] G. Doddington, Automatic evaluation of machine translation quality using n-gram co-occurrence statistics, in: Proceedings of the second international conference on Human Language Technology Research, Morgan Kaufmann Publishers Inc., 2002, pp. 138-145.

[159] S. Banerjee, A. Lavie, METEOR: An automatic metric for MT evaluation with improved correlation with human judgments, in: Proceedings of the acl workshop on intrinsic and extrinsic evaluation measures for machine translation and/or summarization, Vol. 29, 2005, pp. 65-72.

[160] Matthew Snover and Bonnie Dorr and Richard Schwartz and Linnea Micciulla and John Makhoul, A study of translation edit rate with targeted human annotation, in: In Proceedings of Association for Machine Translation in the Americas, 2006, pp. 223-231.

[161] C.-k. Lo, D. Wu, MEANT: an inexpensive, high-accuracy, semiautomatic metric for evaluating translation utility via semantic frames, in: Proceedings of the 49th Annual Meeting of the Association for Computational Linguistics: Human Language TechnologiesVolume 1, Association for Computational Linguistics, 2011, pp. 220229.

[162] C.-k. Lo, P. Dowling, D. Wu, Improving evaluation and optimization of MT systems against MEANT., in: WMT@ EMNLP, 2015, pp. 434-441.

[163] M. Popović, chrF: character n-gram F-score for automatic MT evaluation, in: Proceedings of the Tenth Workshop on Statistical Machine Translation, 2015, pp. 392-395.

[164] M. Popović, chrF deconstructed: beta parameters and n-gram weights, in: Proceedings of the First Conference on Machine Translation: Volume 2, Shared Task Papers, Vol. 2, 2016, pp. 499-504.

[165] M. Popović, chrF++: words helping character n-grams, in: Proceed- 
ings of the Second Conference on Machine Translation, 2017, pp. 612618.

[166] A. Miles, et al., SKOS core: simple knowledge organisation for the web (2005).

[167] D. Nadeau, S. Sekine, A survey of named entity recognition and classification, Lingvisticae Investigationes 30 (1) (2007) 3-26.

[168] P. Koehn, H. Hoang, A. Birch, C. Callison-Burch, M. Federico, N. Bertoldi, B. Cowan, W. Shen, C. Moran, R. Zens, et al., Moses: Open source toolkit for statistical machine translation, in: Proceedings of the 45 th annual meeting of the ACL on interactive poster and demonstration sessions, Association for Computational Linguistics, 2007, pp. 177-180.

[169] R. Navigli, S. P. Ponzetto, Multilingual WSD with just a few lines of code: the BabelNet API, in: Proceedings of the ACL 2012 System Demonstrations, Association for Computational Linguistics, 2012, pp. $67-72$.

[170] P. N. Mendes, M. Jakob, A. Garcia-Silva, C. Bizer, DBpedia Spotlight: shedding light on the web of documents, in: Proceedings of the 7 th international conference on semantic systems, ACM, 2011, pp. 1-8.

[171] K. Cho, B. Van Merriënboer, C. Gulcehre, D. Bahdanau, F. Bougares, H. Schwenk, Y. Bengio, Learning phrase representations using RNN encoder-decoder for statistical machine translation, arXiv preprint arXiv:1406.1078.

[172] P. Monachesi, L. Lemnitzer, K. Simov, Language technology for elearning, in: European Conference on Technology Enhanced Learning, Springer, 2006, pp. 667-672.

[173] A. Gangemi, R. Navigli, P. Velardi, The OntoWordNet Project: extension and axiomatization of conceptual relations in WordNet, in: OTM Confederated International Conferences" On the Move to Meaningful Internet Systems", Springer, 2003, pp. 820-838.

[174] P. Buitelaar, P. Cimiano, B. Magnini, Ontology learning from text: methods, evaluation and applications, Vol. 123, IOS press, 2005.

[175] F. Essalmi, L. J. B. Ayed, Graphical UML view from extended backusnaur form grammars, in: Advanced Learning Technologies, 2006. Sixth International Conference on, IEEE, 2006, pp. 544-546.

[176] R. M. Kaplan, The formal architecture of lexical-functional grammar, J. Inf. Sci. Eng. 5 (4) (1989) 305-322.

[177] J. Gracia, E. Montiel-Ponsoda, P. Cimiano, A. Gómez-Pérez, P. Buitelaar, J. McCrae, Challenges for the multilingual web of data, Web Semantics: Science, Services and Agents on the World Wide Web 11 (2012) 63-71.

[178] J. E. Labra Gayo, D. Kontokostas, S. Auer, Multilingual linked data patterns, Semantic Web 6 (4) (2015) 319-337.

[179] A. Zaveri, A. Rula, A. Maurino, R. Pietrobon, J. Lehmann, S. Auer, Quality assessment for linked data: A survey, Semantic Web 7 (1) (2015) 63-93.

[180] J. Bosque-Gil, J. Gracia, G. Aguado-de Cea, E. Montiel-Ponsoda, Applying the OntoLex Model to a Multilingual Terminological Resource, in: The Semantic Web: ESWC 2015 Satellite Events, Springer, 2015, pp. 283-294.

[181] M. Carpuat, D. Wu, Improving Statistical Machine Translation Using Word Sense Disambiguation., in: EMNLP-CoNLL, Vol. 7, 2007, pp. 6172 .

[182] R. Navigli, Word sense disambiguation: A survey, ACM Computing Surveys (CSUR) 41 (2) (2009) 10.

[183] M. R. Costa-Jussà, M. Farrús, Statistical machine translation enhancements through linguistic levels: A survey, ACM Computing Surveys (CSUR) 46 (3) (2014) 42.

[184] K. Prasad, Principles of Digital Communication System \& Computer Network, Dreamtech Press, 2003.

[185] R. Navigli, S. P. Ponzetto, BabelNet: The automatic construction, evaluation and application of a wide-coverage multilingual semantic network, Artificial Intelligence 193 (2012) 217-250.

[186] O. Bojar, R. Chatterjee, C. Federmann, Y. Graham, B. Haddow, S. Huang, M. Huck, P. Koehn, Q. Liu, V. Logacheva, et al., Findings of the 2017 conference on machine translation (WMT17), in: Proceedings of the Second Conference on Machine Translation, 2017, pp. 169214.

[187] C. D. Manning, Part-of-speech Tagging from 97 to 100: Is It Time for Some Linguistics?, in: Proceedings of the 12th International Conference on Computational Linguistics and Intelligent Text Processing,
2011, pp. 171-189.

[188] S. Legrand, J. Pulido, A hybrid approach to word sense disambiguation: Neural clustering with class labeling (2004).

[189] S. Abburu, A survey on ontology reasoners and comparison, International Journal of Computer Applications 57 (17) (2012) 33-39.

[190] J. Lehmann, Dl-learner: Learning concepts in description logics, J. Mach. Learn. Res. 10 (2009) 2639-2642.

[191] C. Chiarcos, M. Sukhareva, Olia-ontologies of linguistic annotation, Semantic Web 6 (4) (2015) 379-386.

[192] J. McCrae, D. Spohr, P. Cimiano, Linking lexical resources and ontologies on the semantic web with lemon, in: Extended Semantic Web Conference, Springer, 2011, pp. 245-259.

[193] S. Farrar, D. T. Langendoen, A linguistic ontology for the semantic web, GLOT international 7 (3) (2003) 97-100.

[194] S. Hellmann, J. Lehmann, S. Auer, M. Brümmer, Integrating NLP using linked data, in: International Semantic Web Conference, Springer, 2013, pp. 98-113.

[195] D. Moussallem, R. Usbeck, M. Röeder, A.-C. N. Ngomo, MAG: A Multilingual, Knowledge-base Agnostic and Deterministic Entity Linking Approach, in: Proceedings of the Knowledge Capture Conference, ACM, 2017, p. 9.

[196] R. Speck, A.-C. N. Ngomo, Ensemble Learning of Named Entity Recognition Algorithms using Multilayer Perceptron for the Multilingual Web of Data, in: Proceedings of the Knowledge Capture Conference, ACM, 2017, p. 26.

[197] A. Gangemi, A comparison of knowledge extraction tools for the semantic web, in: Extended Semantic Web Conference, Springer, 2013, pp. 351-366.

[198] U. Bojārs, J. G. Breslin, A. Finn, S. Decker, Using the Semantic Web for linking and reusing data across Web 2.0 communities, Web Semantics: Science, Services and Agents on the World Wide Web 6 (1) (2008) 2128.

[199] D. Moussallem, M. A. Sherif, D. Esteves, M. Zampieri, A.-C. N. Ngomo, LIdioms: A Multilingual Linked Idioms Data Set, in: LREC 2018, 2018, p. 7.

[200] J. P. McCrae, M. Arcan, K. Asooja, J. Gracia, P. Buitelaar, P. Cimiano, Domain adaptation for ontology localization, Web Semantics: Science, Services and Agents on the World Wide Web 36 (2016) 23-31.

[201] M. Arcan, S. M. Thomas, D. De Brandt, P. Buitelaar, Translating the FINREP taxonomy using a domain-specific corpus, Machine Translation Summit XIV

[202] M. Arcan, P. Buitelaar, Ontology Label Translation., in: HLT-NAACL, 2013, pp. 40-46.

[203] P. Ristoski, H. Paulheim, Rdf2vec: Rdf graph embeddings for data mining, in: International Semantic Web Conference, Springer, 2016, pp. 498-514.

[204] T. Mikolov, K. Chen, G. Corrado, J. Dean, Efficient estimation of word representations in vector space, arXiv preprint arXiv:1301.3781.

[205] M. Chen, Y. Tian, M. Yang, C. Zaniolo, Multilingual Knowledge Graph Embeddings for Cross-lingual Knowledge Alignment, in: Proceedings of the 26th International Joint Conference on Artificial Intelligence (IJCAI), AAAI Press, 2017, pp. 1-10.

[206] M. Arcan, P. Buitelaar, Translating domain-specific expressions in knowledge bases with neural machine translation, CoRR abs/1709.02184.

[207] Z. Elloumi, H. Blanchon, G. Serasset, L. Besacier, METEOR for Multiple Target Languages using DBnary, in: MT Summit 2015, 2015, pp. 1-11.

[208] C. Servan, A. Bérard, Z. Elloumi, H. Blanchon, L. Besacier, Word2Vec vs DBnary: Augmenting METEOR using Vector Representations or Lexical Resources?, in: COLING 2016, 2016, pp. 1159-1168.

[209] G. Sérasset, Dbnary: Wiktionary as a lemon-based multilingual lexical resource in rdf, Semantic Web 6 (4) (2015) 355-361.

[210] R. Usbeck, et al., AGDISTIS-graph-based disambiguation of named entities using linked data, in: The Semantic Web ISWC 2014, Springer, 2014, pp. 457-471.

[211] A. Moro, F. Cecconi, R. Navigli, Multilingual word sense disambiguation and entity linking for everybody, in: Proceedings of the 2014 International Conference on Posters \& Demonstrations Track-Volume 1272, CEUR-WS. org, 2014, pp. 25-28.

[212] R. Chatterjee, M. Negri, M. Turchi, M. Federico, L. Specia, F. Blain, Guiding neural machine translation decoding with external knowl- 
edge, in: Proceedings of the Second Conference on Machine Translation, 2017, pp. 157-168.

[213] C. Hokamp, Q. Liu, Lexically constrained decoding for sequence generation using grid beam search, in: Proceedings of the 55th Annual Meeting of the Association for Computational Linguistics (Volume 1: Long Papers), Association for Computational Linguistics, 2017, pp. $1535-1546$. 